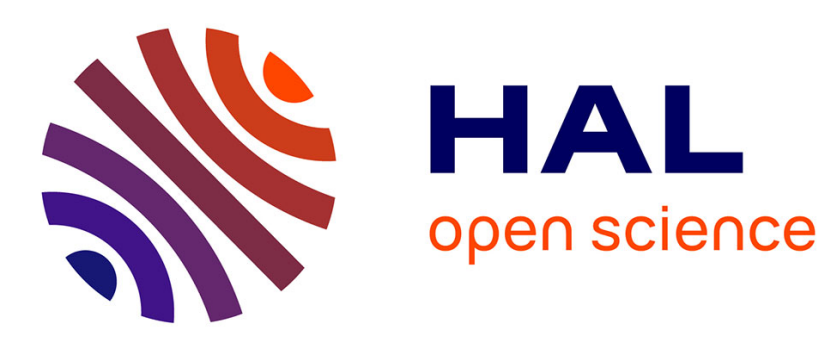

\title{
Mathematical modelling of Acoustic Radiation Force in transient shear wave elastography in the heart
}

Federica Caforio, Sébastien Imperiale

\section{To cite this version:}

Federica Caforio, Sébastien Imperiale. Mathematical modelling of Acoustic Radiation Force in transient shear wave elastography in the heart. ESAIM: Mathematical Modelling and Numerical Analysis, In press. hal-01992069v3

\section{HAL Id: hal-01992069 \\ https://hal.inria.fr/hal-01992069v3}

Submitted on 27 Mar 2020

HAL is a multi-disciplinary open access archive for the deposit and dissemination of scientific research documents, whether they are published or not. The documents may come from teaching and research institutions in France or abroad, or from public or private research centers.
L'archive ouverte pluridisciplinaire HAL, est destinée au dépôt et à la diffusion de documents scientifiques de niveau recherche, publiés ou non, émanant des établissements d'enseignement et de recherche français ou étrangers, des laboratoires publics ou privés. 


\title{
Mathematical modelling of Acoustic Radiation Force in transient shear wave elastography in the heart
}

\author{
Federica Caforio ${ }^{1,2,3}$ and Sébastien Imperiale ${ }^{1,2}$ \\ ${ }^{1}$ Inria, Université Paris-Saclay, France, firstname. lastname@inria.fr \\ ${ }^{2}$ LMS, Ecole Polytechnique, CNRS, Université Paris-Saclay, France \\ ${ }^{3}$ Current affiliation: Computational Cardiology Laboratory, Institute of Biophysics, Medical \\ University of Graz, Austria
}

\begin{abstract}
The aim of this work is to provide a mathematical model and analysis of the excitation and the resulting shear wave propagation in Acoustic Radiation Force (ARF)-based shear wave cardiac elastography. Our approach is based on asymptotic analysis; more precisely, it consists in considering a family of problems, parametrised by a small parameter inversely proportional to the excitation frequency of the probes, the viscosity and the velocity of pressure wave propagation. We derive a simplified model for the expression of the ARF by investigating the limit behaviour of the solution when the small parameter goes to zero. By formal asymptotic analysis - an asymptotic expansion of the solution is used - and energy analysis of the nonlinear elastodynamic problem, we show that the leadingorder term of the expansion is solution of the underlying, incompressible, nonlinear cardiac mechanics. Subsequently, two corrector terms are derived. The first is a fast-oscillating pressure wave generated by the probes, solution of a Helmholtz equation at every time. The second corrector term consists in an elastic field with prescribed divergence, having a function of the first corrector as a source term. This field corresponds to the shear acoustic wave induced by the ARF. We also confirm that, in cardiac mechanics, the presence of viscosity in the model is essential to derive an expression of the shear wave propagation from the ARF, and that this phenomenon is related to the nonlinearity of the partial differential equation.
\end{abstract}

Keywords: Shear Wave Elastography, Acoustic Radiation Force, Asymptotic analysis

\section{Introduction}

Shear acoustic waves remotely induced by the acoustic radiation force (ARF) of a focused ultrasound beam have raised a growing interest in biomedical applications, mainly in elasticity imaging. This is due to the fact that, by measuring the velocity of propagation of the generated shear waves, it is possible 


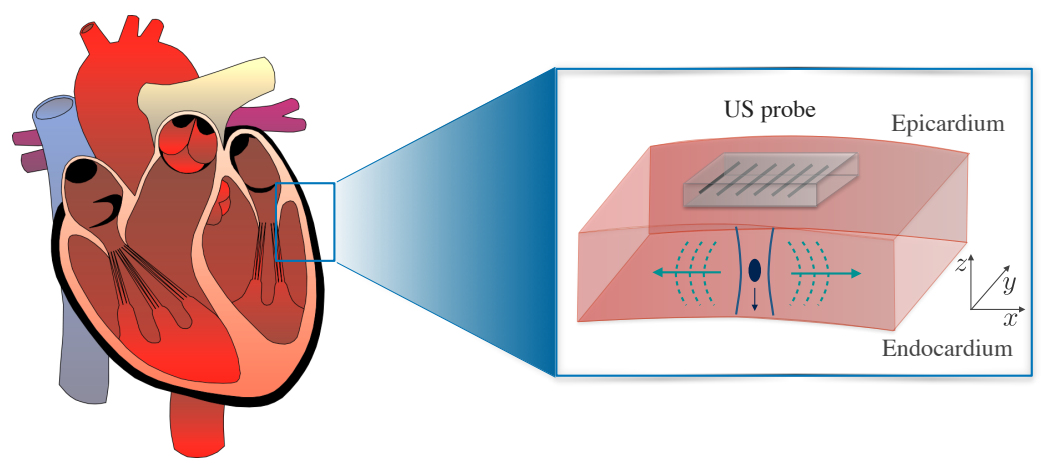

Figure 1: Schematic description of shear wave generation.

to locally assess biomechanical properties highly sensitive to structural changes associated with physiological and pathological processes. The development of the first ARF-based shear wave elastography (SWE) technique dates back to late 1990s and was proposed under the name of Shear Wave Elastography Imaging (SWEI) [1]. Thereafter, several techniques based on impulsive acoustic radiation force were introduced, e.g. Vibro-acoustic Spectography [2], Acoustic Radiation Force Imaging (ARFI) [3], Supersonic Shear Imaging (SSI) [4], Spatially Modulated Ultrasound Radiation Force (SMURF) [5] and Comb-push Ultrasound Shear Elastography (CUSE) [6].

ARF-driven SWE techniques are based on three main steps. First, an acoustic radiation force source is used to remotely induce shear wave propagation in the tissue (Figure 1). ARF is obtained from the emission of a high-intensity acoustic pressure pulse at a specific tissue depth by a conventional ultrasound transducer. Shear motion is then tracked by ultrasound imaging, by emission of low-intensity pulses. Finally, tissue properties are quantitatively estimated by post-processing techniques and inversion algorithms. We recall that in nearly-incompressible soft media, such as biological tissues, the propagation velocity of shear waves $\left(1 \mathrm{~m} \mathrm{~s}^{-1}\right.$ to $\left.10 \mathrm{~m} \mathrm{~s}^{-1}\right)$ is much smaller than the velocity of pressure waves $\left(1500 \mathrm{~m} \mathrm{~s}^{-1}\right)$, and this difference is fundamental for elastography. In more detail, the ARF phenomenon is caused by the transfer of momentum from the focused ultrasound wave to the medium, mainly due to wave absorption $[7,8,9]$. It is generally defined as a time-averaged force applied to a medium by a sound wave. The main orientation of this body force (also known as radiation pressure) is perpendicular to the probe surface. Tissue displacements are first localised close to the focus, then they start propagating in the medium. Particle displacements of these shear waves are oriented parallel to the beam axis. Two relevant advantages of ARF-driven SWE are the highly localised motion and the full attenuation of shear waves in a few wavelengths distance from the focal point of the ultrasonic beam. This induces a narrowing of the induced strain and excludes coupling phenomena at the surface of the tissue. Consequently, the dynamics of the localised motion is only defined by the parameters of acoustic excitation and the mechanical properties of the tissue. Furthermore, it is not necessary to incorporate complicated modelling ingredients (e.g. specific boundary conditions) that take into account far-field interactions to model this phenomenon. This represents a great simplification in reconstruction methods, since the qual- 
ity of reconstruction can be significantly affected by errors in the definition of the boundary conditions [10].

Various models to analyse the physical mechanisms responsible for the ARF and the remote generation of shear waves in water-like media have been proposed. The first theoretical models of shear oscillations remotely induced by radiation force are presented in [1] and [11] . The models are derived in the paraxial regime from the Khokhlov-Zabolotskaya-Kuznetsov (KZK) equation [12]. In that context, we refer to $[13,14]$ for a derivation of an evolution equation for shear waves in a nonlinear soft medium. A substantial contribution in this subject is also due to E. Dontsov and B. B. Guzina. In more detail, they have derived an expression of the body force generated by a high-intensity, focused ultrasound signal, modulated by a low-frequency signal, induced in a Newtonian fluid [15], or an isotropic, viscoelastic, homogeneous solid with heat conduction [16]. To do so, they split the temporal variable into a "fast" (associated with the high-frequency pulse) and "slow" component (related to the ultrasound modulation), they consider that the pressure wave has a specific structure, it is a quasi-plane wave or a focused-beam and they perform a linearisation around an equilibrium state. Moreover, assuming some a-priori properties of the solution, they derive the nonlinear acoustic ARF source term. Furthermore, they have analysed the discrepancy of the solution with respect to the classical solution of the KZK equation, due to the effects of modulation [17]. The role of inhomogeneity and viscosity in the generation of shear waves is analysed in [18], and a theoretical model for shear stress and shear motion in an isotropic solid is presented. It is also proved that an acoustic field in a purely elastic (non-dissipative), homogeneous and nonlinear solid cannot generate shear motion.

Nonetheless, there is still a lack of a complete mathematical analysis of all the phenomena involved in the ARF and the related shear wave generation, in particular when complex soft tissues are considered, such as a fibered muscle tissue. The aim of this work is to partially fill this gap. In this article, we introduce a new methodology to analyse the ARF and provide a new perspective on the understanding and the practical simulation of this phenomenon.

In more detail, the approach adopted is based on two-scale homogenisation techniques - that are standard for the space variable - for the time variable. The use of two time scales is not original for this problem (see [16]). However, our approach relies on an asymptotic ansatz, that leads to a series expansion - related to a small parameter $\varepsilon$ - of the solution, and each term of this expansion is systematically studied. We assume, indeed, that two time variables can be distinguished: one slow, related to the nonlinear mechanics and the shear wave propagation, and a fast one, associated with the pressure wave induced by the ARF excitation. In order to derive a simplified model for the expression of the ARF, we approximate the solution by the first terms of the asymptotic expansion, and we insert this approximation into the elastodynamic equation. Then, we identify and solve the equations proportional to the same order of $\varepsilon$. However, such approach is not sufficient to be conclusive and we need to take into account a thorough energy analysis to derive our results.

Moreover, the results are obtained by considering a family of problems parametrised by $\varepsilon$, that is inversely proportional to the excitation frequency of the probes, the viscosity and the particle velocity of the pressure wave propagation. To our knowledge, this corresponds to the minimal set of assump- 
tions necessary to understand, by asymptotic methods, the ARF phenomenon. By doing so, we avoid the standard simplifying hypotheses on the properties of the emitted pressure waves. For instance, in $[1,11,13,14]$ the expression of the ARF is deduced under the assumption that paraxial approximations are valid. Furthermore, we consider a general constitutive law for hyperelastic, nonlinear soft tissues that can be heterogeneous and anisotropic, and the formulation proposed in this work is valid for media undergoing large deformations, independently from the nature of the prestress. Since one of our applications is the cardiac setting, we also consider the electric activation of the muscle fibres responsible for the heart contraction. Finally, we also derive an original expression of the ARF that is compatible with standard Finite Element (FE) computations, allowing to overcome the standard assumptions that are made on the resulting body force: typically, a Gaussian profile centred around a focalisation point (see $[19,20])$. More precisely, we show that the computation of the ARF source term involves the solution of a scalar Helmholtz problem in the frequency domain at each time instant of the shear wave propagation.

The article is organised as follows. First, some preliminary notions on the elastodynamic problem and our mathematical approach are provided in Section 2. In more detail, the constitutive laws of the medium under consideration are introduced. Then, a family of problems is proposed and a stability estimate is proved. In Section 3 a regular asymptotic expansion of the solution is defined. Moreover, the nonlinear equation for the limit term of the expansion and the equation for the higher-order correctors are given in a general framework. Subsequently, the main contributions of this work are summarised and the results are discussed in Section 4. The methodological approach used to retrieve our formulation is detailed in Section 5. In particular, we derive the equation satisfied by the leading-order term of the expansion, that is associated with the nonlinear heart mechanics, and the governing equations of two corrector terms. The first consists in a fast-oscillating pressure wave excited by the probes, and we show that it is the solution of a Helmholtz equation at every time. The second corrector term is shown to be an elastic field with prescribed divergence, having as a source term a function of the first corrector. This field corresponds to the shear acoustic wave induced by the ARF. A detailed expression of the source term responsible for shear wave propagation is also provided. As a by-product of our analysis we prove that, in prestressed media, the presence of viscosity is essential to produce shear waves with the ARF. Furthermore, they are related to the nonlinearity of the partial differential equation (PDE). Appendix A is devoted to further considerations and detail on some theoretical results.

\section{A 3D nonlinear model for ARF and elastic wave propagation}

Domains and deformation maps. For simplicity of analysis, we consider a smooth, bounded, convex, domain $\Omega_{0} \subset \mathbb{R}^{3}$, that corresponds to some region of the domain under investigation. We consider that forces are imposed at the boundary of the domain

$$
\partial \Omega_{0}=\Gamma_{0} .
$$


Preliminarily, for any observation time $t \in[0, T]$ - where $T$ is the final time of observation - we define the deformation map as a bijective map

$$
\underline{\phi}_{0}(\cdot, t): \bar{\Omega}_{0} \rightarrow \bar{\Omega}(t) \subset \mathbb{R}^{3},
$$

from the reference to the deformed configuration, that reads $\underline{\phi}_{0}: \underline{\xi} \mapsto \underline{x}=\underline{\phi}_{0}(\underline{\xi}, t)$. Then, the limit displacement field $\underline{y}_{0}$ reads $\underline{y}_{0}(\underline{\xi}, t)=\underline{x}-\underline{\xi}=\underline{\phi}_{0}(\underline{\xi}, t)-\underline{\xi}$. Note that we define the deformed configuration only using a limit displacement field (the term limit will be explained later). This field is solution of a nonlinear elastodynamic problem that is independent of the ARF generation process (see Section 3.2.1 for more details, in particular it is assumed to satisfy $J_{0}=1$ and to be smooth).

For the sake of simplicity, we assume that the operators $\underline{\underline{\nabla}} \underline{\nabla}, \Delta$, div, $\underline{\text { div }}$ and $\underline{\underline{H}}$ (Hessian matrix) correspond to differential operators in the reference configuration, and we use the same symbols with the subscript $\underline{x}$ when considering differentiation in the deformed configuration. Moreover, denoting $\underline{\underline{F}}_{0}=\underline{\underline{\nabla}}_{0}$ and $J_{0}=\operatorname{det} \underline{\underline{F}}$, for any smooth function $f$ of $\Omega_{0}$ and $h$ of $\Gamma_{0}$, we have

$$
\int_{\Omega_{0}} f J_{0} \mathrm{~d} \Omega_{0}=\int_{\Omega(t)}\left(f \circ \underline{\phi}_{0}^{-1}\right) \mathrm{d} \Omega
$$

and

$$
\int_{\Gamma_{0}} h J_{0} \underline{\underline{F}}_{0}^{-T} \cdot \underline{n}_{0} \mathrm{~d} \Gamma_{0}=\int_{\Gamma(t)}\left(h \circ \underline{\phi}_{0}^{-1}\right) \underline{n}(t) \mathrm{d} \Gamma \quad \text { with } \quad \underline{n}(t) \circ \underline{\phi}_{0}=\frac{\underline{\underline{F}}_{0}^{-T} \cdot \underline{n}_{0}}{\left|\underline{\underline{F}}_{0}^{-T} \cdot \underline{n}_{0}\right|},
$$

and where $\underline{n}_{0}$ and $\underline{n}(t)$ are the outward unitary normals of $\Omega_{0}$ and $\Omega(t)$ respectively and $\Gamma(t)=\underline{\phi}_{0}\left(\Gamma_{0}\right)$. Henceforth, if not specified, we will implicitly assume the composition with the deformation map $\underline{\phi}_{0}$ when we consider a field in the reference configuration that is defined in the deformed configuration, and conversely for $\phi_{0}^{-1}$.

The fundamental equation of nonlinear elastodynamics. As given in [21], we use the following variational formulation to describe the nonlinear mechanics (in particular it describes the nonlinear cardiac mechanics) in the Lagrangian framework, in absence of external volume forces (e.g. gravity):

Given an admissible functional space $X$, for all $t \in[0, T]$, find $\underline{y}(t) \in X$ such that for all $\underline{w} \in X$

$$
\int_{\Omega_{0}} \rho_{0} \partial_{t}^{2} \underline{y} \cdot \underline{w} \mathrm{~d} \Omega_{0}+\int_{\Omega_{0}} \underline{\underline{\Sigma}}: d_{\underline{y}} \underline{\underline{e}} \cdot \underline{w} \mathrm{~d} \Omega_{0}=\int_{\Gamma_{0}} \underline{\mathrm{t}} \cdot \underline{w} \mathrm{~d} \Gamma_{0}
$$

with vanishing initial conditions $\underline{y}(0)=\underline{0}$ and $\partial_{t} \underline{y}(0)=\underline{0}$. Defining the deformation gradient $\underline{\underline{F}}=\underline{\underline{\mathbb{1}}}+\underline{\underline{\nabla}} \underline{y}$, we recall that $\underline{\underline{e}}$ is the Green-Lagrange strain tensor and reads

$$
\underline{\underline{e}}=\frac{1}{2}\left(\underline{\underline{F}}^{T} \underline{\underline{F}}-\underline{\underline{\mathbb{1}}}\right)=\frac{1}{2}\left(\underline{\underline{\nabla}} \underline{y}+(\underline{\underline{\nabla}} \underline{y})^{T}+(\underline{\underline{\nabla}} \underline{y})^{T} \cdot \underline{\underline{\nabla}} \underline{y}\right) .
$$

In (2.2), $d_{\underline{y}} \underline{\underline{e}}$ is the derivative of the Green-Lagrange tensor with respect to the displacement, and it is given by

$$
d_{\underline{y}} \underline{\underline{e}} \cdot \underline{w}=\frac{1}{2}\left((\underline{\underline{\nabla}} \underline{w})^{T} \cdot \underline{\underline{F}}+\underline{\underline{F}}^{T} \cdot \underline{\underline{\nabla}} \underline{w}\right)
$$


Moreover, $\underline{\underline{\Sigma}}$ is the Second Piola-Kirchhoff stress tensor accounting for total stress, and $\underline{\mathrm{t}}$ (in $\mathrm{kg} \mathrm{m}^{-1} \mathrm{~s}^{-2}$ ) accounts for the Lagrangian counterpart to the surface excitation on the boundary, due to the piezoelectric probes. We refer the reader to $[22,23,24]$ for supporting material on continuum mechanics and nonlinear elasticity. The operator "." corresponds to scalar product, whereas ":" stands for dyadic product. Note also that, since we consider viscosity in the constitutive law, we must introduce the following notation

$$
\underline{\underline{\dot{e}}}=\partial_{t} \underline{\underline{e}}=\frac{1}{2}\left(\left(\underline{\underline{\nabla}} \partial_{t} \underline{y}\right)^{T} \cdot \underline{\underline{F}}+\underline{\underline{F}}^{T} \cdot \underline{\underline{\nabla}} \partial_{t} \underline{y}\right)=d_{\underline{y}} \underline{\underline{e}} \cdot \partial_{t} \underline{y} .
$$

Since units matter in our analysis, we already specify that $y$ is in $\mathrm{m}$ and $t$ is in s. Note that, by their very definitions, $\underline{\underline{e}}$ is dimensionless, whereas $\underline{\underline{e}}$ is in $\mathrm{s}^{-1}$.

\subsection{Constitutive assumptions and energy identity}

Definition of stresses using potentials. For cardiac muscle tissue, it is standard to consider a constitutive law that is composed of two contributions, accounting for passive and active stress [21]. Therefore, we define

$$
\underline{\underline{\Sigma}}=\underline{\underline{\Sigma}}^{P}+\kappa_{a} \underline{\underline{\Sigma}}^{A}
$$

where $\kappa_{a}$ is a strictly positive parameter (in $\mathrm{kg} \mathrm{m}^{-1} \mathrm{~s}^{-2}$ ). As far as the passive term is concerned, we consider a visco-hyperelastic medium, therefore we can express

$$
\underline{\underline{\Sigma}}^{P}=\frac{\partial W}{\partial \underline{\underline{e}}}+\zeta \frac{\partial V}{\partial \underline{\underline{\dot{e}}}}
$$

where $\zeta$ is a strictly positive parameter (in $\mathrm{kg} \mathrm{m}^{-1} \mathrm{~s}^{-1}$ ), $W$ is the hyperelastic potential and $V$ is the viscous pseudo-potential. In cardiac modelling (see $[21,25]$ ) it is standard to take into account a hyperelastic potential composed of a term accounting for the anisotropy induced by the presence of the muscle fibres and a penalisation term related to near-incompressibility (NI). More precisely, we set

$$
W=\kappa_{s} W^{\mathrm{TI}}+\kappa W^{\mathrm{NI}}
$$

where $\kappa$ and $\kappa_{s}$ are strictly positive parameters in $\mathrm{kg} \mathrm{m}^{-1} \mathrm{~s}^{-2}$ ( $\kappa$ is the so-called bulk modulus). The dimensionless potential associated with near-incompressibility reads

$$
W^{\mathrm{NI}}(\underline{\underline{e}}):=\frac{1}{4}\left(\left(J^{2}-1\right)-\log \left(J^{2}\right)\right)
$$

where $J=\operatorname{det} \underline{\underline{F}}$ stands for the Jacobian of the deformation. Given some non-dimensionalised strictly positive parameters $\left\{\hat{\kappa}_{i}\right\}$ and $\left\{\hat{\eta}_{i}\right\}$, the potential - that is dimensionless as well - associated with the transversely isotropic constitutive law reads

$$
W^{\mathrm{TI}}(\underline{\underline{e}}):=\hat{\kappa}_{1} e^{\hat{\eta}_{1}\left(I_{1}-3\right)^{2}}+\hat{\kappa}_{2} e^{\hat{\eta}_{2}\left(I_{4}-1\right)^{2}}+\hat{\kappa}_{3}\left(I_{2}-2 \log \left(J^{2}\right)-3\right),
$$


where, defining the right Cauchy-Green strain tensor $\underline{\underline{C}}=\underline{\underline{F}}^{T} \cdot \underline{\underline{F}}=\underline{\underline{\mathbb{1}}}+2 \underline{\underline{e}}$, we have

$$
I_{1}=\operatorname{tr}(\underline{\underline{C}}), \quad I_{2}=|\operatorname{adj} \underline{\underline{F}}|^{2}, \quad I_{4}=\underline{\tau} \cdot \underline{\underline{C}} \cdot \underline{\tau},
$$

where $\underline{\tau}(\underline{\xi})$ is the unit vector associated with the - space-dependent - fibre direction. Note that the norm operator $|\cdot|$ denotes the euclidean norm in $\mathbb{R}^{d}$ or the Frobenius norm $\mathbb{R}^{d \times d}$, accordingly. The potential $W^{\mathrm{TI}}$ is defined as a combination of a transversely isotropic exponential law - which shares some similarities with the law proposed in [26] - endowed with a penalisation term for incompressibility and a linear, isotropic term inspired by the well-known Ciarlet-Geymonat constitutive law [27]. In particular, the linear term is inserted in order to ensure overall stability of the system, whereas the exponential component dominates in large deformations. We refer the reader to [28, Chapter 1] for further detail on the choice and the properties of this constitutive law. Finally, the viscous pseudo-potential - in $\mathrm{s}^{-2}-$ reads

$$
V(\underline{\underline{e}}, \underline{\underline{e}}):=\frac{1}{2} \operatorname{tr}\left(\underline{\underline{C}}^{-1} \cdot \underline{\underline{\dot{e}}}\right)^{2}=\frac{1}{2}\left(\partial_{t} \log \left(J^{2}\right)\right)^{2} .
$$

We emphasise that the expression of $V$ in Eq. (2.9) only involves $J$, hence viscosity acts as a selective damping that has the important effect of damping linearised pressure waves, as we will see later. Our analysis could be extended to more complex viscosity terms, but this would not change the main physical phenomenon that we want to illustrate. If we define

$$
\underline{\underline{\Sigma}}^{\mathrm{TI}}:=\frac{\partial W^{\mathrm{TI}}}{\partial \underline{\underline{e}}}, \quad \underline{\underline{\Sigma}} \mathrm{NI}:=\frac{\partial W^{\mathrm{NI}}}{\partial \underline{\underline{e}}}=\frac{1}{2}\left(J^{2}-1\right) \underline{\underline{C}}^{-1} \quad \text { and } \quad \underline{\underline{\Sigma}}^{\mathrm{V}}:=\frac{\partial V}{\partial \underline{\underline{\dot{e}}}}=\operatorname{tr}\left(\underline{\underline{C}}^{-1} \cdot \underline{\underline{\dot{e}}}\right) \underline{\underline{C}}^{-1},
$$

then Eq. (2.4) can be recast as $\underline{\underline{\Sigma}}^{P}=\kappa_{s} \underline{\underline{\Sigma}}^{\mathrm{TI}}+\kappa \underline{\underline{\Sigma}}^{\mathrm{NI}}+\zeta \underline{\underline{\Sigma}}^{\mathrm{V}}$. For the active term $\underline{\underline{\Sigma}}^{A}$, we define a simple constitutive law, and we introduce a one-dimensional stress $\sigma_{a}$ in the fibre direction that is dimensionless and is a given, smooth, space and time-dependent function. Namely, we set

$$
\underline{\underline{\Sigma}}^{A}=\sigma_{a} \underline{\tau} \otimes \underline{\tau} \text {. }
$$

Note that $\underline{\underline{\Sigma}}^{A}$ does not depend on the displacement $\underline{y}$ and represents a source term for Problem (2.2). Finally, the other source term of the problem, namely $\underline{\mathrm{t}}$, is described in detail later.

Energy identity. It is standard to show that a smooth solution of Eq. (2.2) satisfies the following energy identity (obtained by simply using $\partial_{t} \underline{y}$ as a test function in Eq. (2.2) and integrating over space and time)

$$
\mathcal{E}^{t o t}(t)+\int_{0}^{t} \mathcal{P}^{\mathrm{V}} \mathrm{d} s=\int_{0}^{t} \mathcal{P}^{e x t} \mathrm{~d} s+\int_{0}^{t} \mathcal{P}^{A} \mathrm{~d} s
$$

where the total energy $\mathcal{E}^{\text {tot }}$ reads

$$
\mathcal{E}^{t o t}=\mathcal{E}^{\mathcal{K}}+\mathcal{E}^{\mathrm{TI}}+\mathcal{E}^{\mathrm{NI}}
$$

and the kinetic and potential energy associated with the hyperelastic term and near-incompressibility are given by

$$
\mathcal{E}^{\mathcal{K}}=\frac{\rho_{0}}{2} \int_{\Omega_{0}}\left|\partial_{t} \underline{y}\right|^{2} \mathrm{~d} \Omega_{0}, \quad \mathcal{E}^{\mathrm{TI}}=\kappa_{s} \int_{\Omega_{0}} W^{\mathrm{TI}}(\underline{\underline{e}}) \mathrm{d} \Omega_{0}, \quad \mathcal{E}^{\mathrm{NI}}=\kappa \int_{\Omega_{0}} W^{\mathrm{NI}}(\underline{\underline{e}}) \mathrm{d} \Omega_{0},
$$


whereas the viscous losses and the source terms are given by

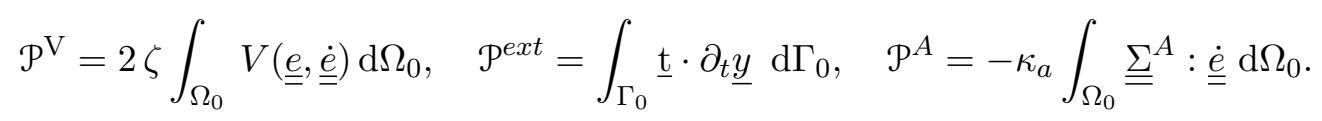

Note that to obtain the energy identity we have used the property $\frac{\partial V}{\partial \underline{\underline{e}}}: \underline{\underline{\dot{e}}}=2 V$, due to Eq. (2.9).

\subsection{Non-dimensionalisation}

In nearly-incompressible soft media, such as biological tissues, the propagation velocity of shear waves $\left(1 \mathrm{~m} \mathrm{~s}^{-1}\right.$ to $\left.10 \mathrm{~m} \mathrm{~s}^{-1}\right)$ is much smaller than the velocity of pressure waves $\left(1500 \mathrm{~m} \mathrm{~s}^{-1}\right)$. Our objective is to consider a family of problems parametrised by a small parameter $\varepsilon$, representing the ratio of the shear wave velocity and pressure wave velocity. To do so, we need to first introduce a non-dimensionalised version of the elastodynamic equations given Section 2.1 above. We define the rescaled displacement (a non-dimensionalised vector field) as

$$
\underline{\hat{y}}(\underline{\xi}, t):=\mathcal{L}^{-1} \underline{y}(\mathcal{L} \underline{\xi}, \mathcal{T} t),
$$

where $\mathcal{L}$ is a typical space scale (typically of the order of the diameter of the domain) and $\mathcal{T}$ a typical time scale of the phenomena under observation. The field $\underline{\hat{y}}(\underline{\xi}, t)$ is defined on a rescaled domain in space - typically with diameter of the order of unity and still denoted $\Omega_{0}$ - and in time - typically of order one and still denoted $[0, T]$. The rescaled parameters $\mathcal{L}$ and $\mathcal{T}$ are set later. Now observe that one has

$$
\left.\underline{\underline{\nabla}} \underline{\hat{y}}(\underline{\xi}, t)=\underline{\nabla} \underline{y}(\mathcal{L} \underline{\xi}, \mathcal{T} t) \quad \text { and } \quad \partial_{t} \underline{\hat{y}} \underline{\xi}, t\right)=\mathcal{T}^{-1} \partial_{t} \underline{y}(\mathcal{L} \underline{\xi}, \mathcal{T} t) .
$$

Therefore, it is straightforward to deduce from (2.12) a dimensionless energy identity for the rescaled displacement. For the sake of simplicity, from now on we drop the $\hat{\imath}$ notation used for the unknown displacement field and all related mechanical quantities. The non-dimensionalised energy relation reads

$$
\begin{aligned}
\frac{\hat{\rho}_{0}}{2} \int_{\Omega_{0}}\left|\partial_{t} \underline{y}\right|^{2} \mathrm{~d} \Omega_{0}+\frac{\kappa}{\kappa_{s}} \int_{\Omega_{0}} W^{\mathrm{NI}}(\underline{\underline{e}}) \mathrm{d} \Omega_{0} & +\int_{\Omega_{0}} W^{\mathrm{TI}}(\underline{\underline{e}}) \mathrm{d} \Omega_{0}+2 \hat{z} \int_{0}^{t} \int_{\Omega_{0}} V(\underline{\underline{e}}, \underline{\underline{e}}) \mathrm{d} \Omega_{0} \mathrm{~d} s \\
& =\frac{1}{\kappa_{s}} \int_{0}^{t} \int_{\Gamma_{0}} \underline{\mathrm{t}} \cdot \partial_{t} \underline{y} \mathrm{~d} \Gamma_{0} \mathrm{~d} s-\frac{\kappa_{a}}{\kappa_{s}} \int_{0}^{t} \int_{\Omega_{0}} \underline{\Sigma}^{A}: \underline{\underline{e}} \mathrm{~d} \Omega_{0} \mathrm{~d} s
\end{aligned}
$$

with

$$
\hat{\rho}_{0}=\frac{\mathcal{L}^{2} \rho_{0}}{\mathcal{T}^{2} \kappa_{s}} \quad \text { and } \quad \hat{z}=\frac{\zeta}{\mathcal{T} \kappa_{s}} .
$$

From Eq. (2.14) one can deduce a non-dimensionalised version of the weak formulation (2.2) (such formulation being rather straightforwardly obtained, we omit it). Moreover, as we shall see next, the identity (2.14) has the adequate structure in order to compare various contributions and therefore to introduce the family of problems mentioned before. 


\subsection{Definition of a parametric family of problems}

Scaling choices. The family of problems is constructed comparing the parameters $\hat{\rho}_{0}, \kappa / \kappa_{s}, \hat{z}$ and $\kappa_{a} / \kappa_{s}$. In the context of this work (shear wave propagation in soft tissues) we choose

$$
\mathcal{L}=10^{-2} \mathrm{~m} \quad \text { and } \quad \mathcal{T}=10^{-2} \mathrm{~s},
$$

since our main focus is the propagation of shear waves $\left(1 \mathrm{~m} \mathrm{~s}^{-1}\right.$ to $\left.10 \mathrm{~m} \mathrm{~s}^{-1}\right)$ in a rectangular slab of an organ (e.g. in some volume of $6 \mathrm{~cm} \times 6 \mathrm{~cm} \times 2 \mathrm{~cm}$ of the left ventricle of the heart [29]). To model the myocardial tissue, in accordance with $[19,28,29,30,26]$ we choose

$$
\kappa \sim 10^{8} \mathrm{~kg} \mathrm{~m}^{-1} \mathrm{~s}^{-2}, \quad \kappa_{s}=10^{3} \mathrm{~kg} \mathrm{~m}^{-1} \mathrm{~s}^{-2},
$$

Therefore we retrieve that $\hat{\rho}_{0} \sim 1$ and $\kappa / \kappa_{s} \sim 10^{5}$, so that we can define the dimensionless small positive parameter $\varepsilon$ as

$$
\varepsilon=\sqrt{\frac{\kappa_{s}}{\kappa}}
$$

where $\frac{\kappa_{s}}{\kappa}$ is commensurate to the ratio between shear and pressure wave velocities. We come now to the scaling choice for the parameter $\hat{\zeta}$. Experimental evidence suggests that pressure waves are also localised, i.e. they are strongly attenuated after a few centimetres, hence we hypothesise that the viscosity coefficient should be strong enough to damp waves that propagate at a high velocity (proportional here to $\varepsilon^{-1}$ ). This naturally leads to the choice

$$
\hat{z}=\varepsilon^{-1} \hat{\zeta}
$$

where $\hat{\zeta}$ is a normalised strictly positive parameter. It turns out that this choice also appears naturally in the details of the asymptotic analysis of the parametric family of problems that we give below. Indeed, if $\hat{z} \sim \varepsilon^{-2}$, then pressure waves do not propagate (the problem degenerates into a parabolic problem). On the contrary, if one chooses $\hat{z} \sim 1$, then pressure waves propagate without attenuation (which is in contradiction with observations). Finally, and less importantly, we set $\kappa_{a}=\kappa_{s}$ (this choice is always possible by simply changing the value of $\sigma_{a}$ ), i.e. we assume that active stresses are much smaller than stresses induced by the near-incompressibility potential.

Definition of the boundary source term. Our asymptotic analysis of the ARF relies on the assumption that the source term - generated by the piezoelectric sensors - is a high-frequency pressure term. In particular, the wavelength of the propagating pressure waves should be rescaled, hence we set the ultrasound frequency proportional to $\varepsilon^{-1}$. In more detail, we assume that the source term satisfies

$$
\frac{1}{\kappa_{s}} \underline{\mathrm{t}}(\underline{\xi}, t):=\underline{\mathrm{t}}_{\varepsilon}=g(\underline{\xi}, t, t / \varepsilon) J \underline{\underline{F}}^{-T} \cdot \underline{n}_{0},
$$

where $\underline{n}_{0}$ is the outward unitary normal and $g(\underline{\xi}, t, \tau)$ is a dimensionless smooth space and time-dependent function, periodic in $\tau$ with zero average and period $2 \pi / \hat{\omega}$, where $\hat{\omega}>0$ is a given normalised frequency. The remaining terms in (2.16) account for the assumption that the source term is normal to the surface 
in the deformed configuration (see Eq. (2.1)).

Definition of a family of problems. For any given $\varepsilon$ small enough, we look for the solution $\underline{y}_{\varepsilon}$ of the weak formulation deduced from Eqs. (2.2) and (2.14) with the scaling choice done above. This formulation reads:

Given an admissible displacement space $\mathcal{X}$, find, for all $t \in[0, T]$, a function $\underline{y}_{\varepsilon}(t) \in \mathcal{X}$ such that for all $\underline{w} \in X$

$$
\begin{aligned}
& \hat{\rho}_{0} \int_{\Omega_{0}} \partial_{t}^{2} \underline{y}_{\varepsilon} \cdot \underline{w} \mathrm{~d} \Omega_{0}+\int_{\Omega_{0}}\left(\underline{\underline{\Sigma}}_{\varepsilon}^{\mathrm{TI}}+\varepsilon^{-2} \underline{\underline{\Sigma}}_{\varepsilon}^{\mathrm{NI}}+\hat{\zeta} \varepsilon^{-1} \underline{\underline{\Sigma}}_{\varepsilon}^{\mathrm{V}}\right): d_{\underline{y}} \underline{\underline{e}} \cdot \underline{w} \mathrm{~d} \Omega_{0} \\
&=-\int_{\Omega_{0}} \underline{\underline{\Sigma}}^{A}: d_{\underline{\underline{y}}} \underline{\underline{e}} \cdot \underline{w} \mathrm{~d} \Omega_{0}+\int_{\Gamma_{0}} \underline{\mathrm{t}}_{\varepsilon} \cdot \underline{w} \mathrm{~d} \Gamma_{0},
\end{aligned}
$$

with vanishing initial conditions $\underline{y}_{\varepsilon}(0)=\underline{0}$ and $\partial_{t} \underline{y}_{\varepsilon}(0)=\underline{0}$. In Eq. (2.17) we have

$$
\underline{\underline{\Sigma}}_{\varepsilon}^{\mathrm{TI}}=\frac{\partial W^{\mathrm{TI}}}{\partial \underline{\underline{e}}}\left(\underline{\underline{e}}_{\varepsilon}\right), \quad \underline{\underline{\Sigma}}_{\varepsilon}^{\mathrm{NI}}=\frac{\partial W^{\mathrm{NI}}}{\partial \underline{\underline{e}}}\left(\underline{\underline{e}}_{\varepsilon}\right), \quad \underline{\underline{\Sigma}}_{\varepsilon}^{\mathrm{V}}=\frac{\partial V}{\partial \underline{\underline{\dot{e}}}}\left(\underline{\underline{e}}_{\varepsilon}, \underline{\underline{e}}_{\varepsilon}\right) .
$$

with $\underline{e}_{\varepsilon}$ the Green-Lagrange strain tensor associated with the displacement $\underline{y}_{\varepsilon}$. The analysis of existence, uniqueness and regularity of solutions of (2.17) is a difficult question that we avoid by stating the following assumption.

Assumption 2.1. For all $\varepsilon>0$ sufficiently small, there exists a unique smooth solution $\underline{y}_{\varepsilon}$ in $\bar{\Omega} \times[0, T]$ to Eq. (2.17) satisfying

$$
J_{\varepsilon}=\operatorname{det} \underline{\underline{F}}_{\varepsilon}=\operatorname{det}\left(\underline{\underline{\mathbb{1}}}+\underline{\underline{\nabla}} \underline{y}_{\varepsilon}\right)>0 .
$$

Stability estimates. Using the same approach adopted to obtain the energy identity (2.14) one can retrieve some energy estimates allowing to derive some interesting properties of the solution $\underline{y}_{\varepsilon}$. Choosing the actual velocity $\partial_{t} \underline{y}_{\varepsilon}$ as a test function in (2.17), we obtain an energy balance that we do not specify here but refer the reader to Appendix A for more details. The energy balance is used in Appendix A to prove the following stability result.

Lemma 2.1. There exists a scalar $C>0$ such that for all $\varepsilon$ small enough

$$
\begin{aligned}
\int_{\Omega_{0}}\left|\partial_{t} \underline{y}_{\varepsilon}\right|^{2} \mathrm{~d} \Omega_{0}+\varepsilon^{-2} \int_{\Omega_{0}}\left(\left(J_{\varepsilon}^{2}-1\right)-\log \left(J_{\varepsilon}^{2}\right)\right) \mathrm{d} \Omega_{0} & \\
& +\varepsilon^{-1} \int_{0}^{t} \int_{\Omega_{0}}\left|\operatorname{tr}\left(\underline{\underline{C}}_{\varepsilon}^{-1} \cdot \underline{\underline{e}}_{\varepsilon}\right)\right|^{2} \mathrm{~d} \Omega_{0} \mathrm{~d} s \leq C \quad \forall t \in[0, T],
\end{aligned}
$$

where $\underline{\underline{C}}_{\varepsilon}=\underline{\underline{F}}_{\varepsilon}^{T} \cdot \underline{\underline{F}}_{\varepsilon}$. 


\section{Asymptotic expansion of the solution}

\subsection{Definition of a truncated power series expansion}

We look for a regular asymptotic expansion of the solution of Eq. (2.17), i.e. we assume that $\underline{y}_{\varepsilon}$ can be written using a truncated power series expansion in $\varepsilon$. More precisely, we assume that there exists an integer $N \geq 4$ such that the solution $\underline{y}_{\varepsilon}$ can be decomposed, for all $\varepsilon$ sufficiently small, as

$$
\underline{y}_{\varepsilon}(\underline{\xi}, t)=\sum_{i=0}^{N} \varepsilon^{i} \underline{y}_{i}(\underline{\xi}, t, t / \varepsilon)+\varepsilon^{N} \underline{r}_{\varepsilon}, \quad \forall \underline{\xi} \in \Omega_{0}, \forall t \in[0, T],
$$

with $\underline{r}_{\varepsilon}$ smooth and $\lim _{\varepsilon \rightarrow 0}\left\|\underline{r}_{\varepsilon}\right\|=0$ for an adequate norm $\|\cdot\|$. We assume that each term $\underline{y}_{i}(\underline{\xi}, t, \tau)$ of the sum is periodic in $\tau \in \mathbb{R}$, with period $2 \pi / \hat{\omega}$ (this is suggested by the structure of the source term). Furthermore, we assume that all the components $\underline{y}_{i}$ inherit the regularity properties - in $\underline{\xi}$ and $t-$ of $\underline{y}_{\varepsilon}$ and are regular enough in $\tau$ (hence all $\partial_{\tau} \underline{y}_{i}$ are periodic in $\tau$ as well).

The idea is to approximate the solution $\underline{y}_{\varepsilon}$ by the first terms of expansion (3.1). By doing so, we recover the limit (asymptotic) behaviour of the solution $y_{\varepsilon}$ for $\varepsilon \rightarrow 0$. In practice, we substitute the expression of $\underline{y}_{\varepsilon}$ presented in Eq. (3.1) in Problem (2.17). To do so, all mechanical quantities must be rewritten in series form, accordingly. Furthermore, we shall use the following expansion of the second derivative in time for each term in Eq. (3.1):

$$
\partial_{t}^{2}\left(\underline{y}_{i}(\underline{\xi}, t, t / \varepsilon)\right)=\left(\varepsilon^{-2} \partial_{\tau}^{2} \underline{y}_{i}+2 \varepsilon^{-1} \partial_{t \tau}^{2} \underline{y}_{i}+\varepsilon^{0} \partial_{t}^{2} \underline{y}_{i}\right)(\underline{\xi}, t, t / \varepsilon) .
$$

Once all the terms of the expansion are obtained and inserted in Eq. (2.17), the equations proportional to the same order of $\varepsilon$ are identified. Note that this approach represents an application of a methodology known as the two-scale homogenisation, which is standard in the spatial domain [31].

In what follows, we show that the ARF is a nonlinear phenomenon that occurs at a higher order of approximation. More precisely, we show that the leading-order term $\underline{y}_{0}$ in the asymptotic expansion (3.1) corresponds to the underlying nonlinear mechanical behaviour of the solution. Then, the first two correctors are analysed. The first corrector is zero, whereas the second one $\underline{y}_{2}$ can be decomposed into two contributions:

- a fast-oscillating pressure wave generated by the piezoelectric probes, solution of a Helmholtz equation at every time;

- a slow-varying elastic field with prescribed divergence, solution of a linear equation having as a source term a quadratic function of the first corrector. This field corresponds to the shear acoustic wave induced by the ARF (and it is divergence-free in case of no prestress). 


\subsection{The incompressible, nonlinear heart mechanics and its linearisation}

In Shear Wave Elastography, the propagation of elastic waves in a prestressed soft medium like the myocardium can be modelled as a perturbation of the underlying nonlinear incompressible heart mechanics. Therefore, we expect the limit term of our expansion to be the solution of this nonlinear problem. Henceforth, we formulate the nonlinear problem and its linearisation that govern some of the terms in the asymptotic expansion (3.1). More precisely, we will show that the perturbation associated with the shear wave propagation satisfies an equation that can be recovered by linearisation of Eq. (2.17) around a reference, time-dependent deformation state. The well-posedness properties of the two problems considered (existence/uniqueness/regularity of solutions) are not analysed but assumed instead.

\subsubsection{Nonlinear mechanical model of heart deformation.}

As it has been already mentioned, we show in what follows that the limit term $\underline{y}_{0}$ in the Ansatz (3.1) is solution of the following nonlinear, incompressible cardiac mechanics independent of the fast time variable $\tau$. Given an adequate functional space $\mathcal{L}$ :

For all $t \in[0, T]$, find $\left(\underline{y}_{0}(t), \mathrm{p}_{0}(t)\right) \in \mathcal{X} \times \mathcal{L}$ such that for all $\underline{w} \in \mathcal{X}$

$$
\hat{\rho}_{0} \int_{\Omega_{0}} \partial_{t}^{2} \underline{y}_{0} \cdot \underline{w} \mathrm{~d} \Omega_{0}+\int_{\Omega_{0}} \mathrm{p}_{0} \underline{\underline{C}}_{0}^{-1}: \underline{\underline{e}}_{0}(\underline{w}) \mathrm{d} \Omega_{0}+\int_{\Omega_{0}} \underline{\underline{\Sigma}}_{0}^{\mathrm{TI}}: \underline{\underline{e}}_{0}(\underline{w}) \mathrm{d} \Omega_{0}=-\int_{\Omega_{0}} \underline{\underline{\Sigma}}^{A}: \underline{\underline{e}}_{0}(\underline{w}) \mathrm{d} \Omega_{0},
$$

satisfying $J_{0}=1$ and with initial conditions $\underline{y}_{0}(0)=\underline{0}$ and $\partial_{t} \underline{y}_{0}(0)=\underline{0}$ and where $\mathrm{p}_{0}$ is a Lagrange multiplier to enforce incompressibility and

$$
\underline{\underline{C}}_{0}=\underline{\underline{F}}_{0}^{T} \cdot \underline{\underline{F}}_{0}, \quad \underline{\underline{e}}_{0}(\underline{w})=d_{\underline{y}_{0}} \underline{\underline{e}}_{0} \cdot \underline{w}=\frac{1}{2}\left(\underline{\underline{F}}_{0}^{T} \cdot \underline{\underline{\nabla}} \underline{w}+(\underline{\underline{\nabla}} \underline{w})^{T} \cdot \underline{\underline{F}}_{0}\right)
$$

whereas

$$
\underline{\underline{\Sigma}}_{0}^{\mathrm{TI}}=\frac{\partial W^{\mathrm{TI}}}{\partial \underline{\underline{e}}}\left(\underline{\underline{e}}_{0}\right) \quad \text { with } \quad \underline{\underline{e}}_{0}=\frac{1}{2}\left(\underline{\underline{F}}_{0}^{T} \underline{\underline{F}}_{0}-\underline{\underline{\mathbb{1}}}\right) .
$$

Note that a detailed expression of $\underline{\underline{\Sigma}}_{0}^{\mathrm{TI}}$ is given in Appendix B. Typically, the space $\mathcal{L}$ is a space of zero-average scalar functions. The analysis of the existence and uniqueness of solution to Eq. (3.2) is a well-known difficult problem; as a consequence, we will state the following assumption.

Assumption 3.1. There exists a unique smooth couple of function $\left(\underline{y}_{0}, \mathrm{p}_{0}\right)$ solution to Eq. (3.2).

\subsubsection{Linearised problem for the elastic perturbation.}

The linearisation of (2.17) around the time-dependent solution $\underline{y}_{0}(t)$ leads to the following linear problem: Given admissible functional spaces for the linearised problem $\bar{X}_{\ell}$ and $\mathcal{L}_{\ell}$, find, for all $t \in[0, T]$, a couple 
$(\underline{\tilde{y}}(t), \tilde{\mathrm{p}}(t)) \in X_{\ell} \times \mathcal{L}_{\ell}$ such that for all $\underline{w} \in X_{\ell}$ and for all $q \in \mathcal{L}_{\ell}$

$$
\left\{\begin{array}{l}
\hat{\rho}_{0} \int_{\Omega_{0}} \partial_{t}^{2} \underline{\tilde{y}} \cdot \underline{w} \mathrm{~d} \Omega_{0}+\int_{\Omega_{0}} \tilde{\mathrm{p}} \underline{\underline{C}}_{0}^{-1}: \underline{\underline{e}}_{0}(\underline{w}) \mathrm{d} \Omega_{0}+a(\underline{\tilde{y}}, \underline{w})=s(t, \underline{w}), \\
\int_{\Omega_{0}} \underline{\underline{C}}_{0}^{-1}: \underline{\underline{e}}_{0}(\underline{\tilde{y}}) q \mathrm{~d} \Omega_{0}=r(t, q),
\end{array}\right.
$$

with initial conditions $\underline{\tilde{y}}(0)=\underline{0}$ and $\partial_{t} \underline{\tilde{y}}(0)=\underline{0}$, where $s(t, \cdot)$ and $r(t, \cdot)$ are some linear forms yet unspecified, and where

$$
\begin{aligned}
a(\underline{\tilde{y}}, \underline{w})= & \int_{\Omega_{0}} \underline{\underline{e}}_{0}(\underline{\tilde{y}}): \underline{\underline{\mathbf{A}}}_{0}: \underline{\underline{e}}_{0}(\underline{w}) \mathrm{d} \Omega_{0}-\int_{\Omega_{0}} \mathrm{p}_{0} \underline{\underline{\nabla}} \underline{\tilde{y}} \cdot \underline{\underline{F}}_{0}^{-1}: \underline{\underline{\nabla}} \underline{w} \cdot \underline{\underline{F}}_{0}^{-1} \mathrm{~d} \Omega_{0} \\
& +\int_{\Omega_{0}}\left(\underline{\underline{\Sigma}}_{0}^{\mathrm{TI}}+\underline{\underline{\Sigma}}^{A}+\mathrm{p}_{0} \underline{\underline{C}}_{0}^{-1}\right):(\underline{\underline{\nabla}} \underline{\tilde{y}})^{T} \cdot \underline{\underline{\nabla}} \underline{w} \mathrm{~d} \Omega_{0},
\end{aligned}
$$

the unknown $\tilde{\mathrm{p}}$ being a Lagrange multiplier enforcing incompressibility. $\underline{\underline{\mathbf{A}}}_{0}$ is a fourth-order tensor corresponding to the derivative of the second Piola-Kirchoff stress tensor $\underline{\underline{\Sigma}}^{\mathrm{TI}}$ with respect to the GreenLagrange strain tensor $\underline{\underline{e}}$, evaluated at $\underline{y}_{0}$. We refer the reader to Appendix B for the detailed expression of the tensor $\underline{\underline{\mathbf{A}}}_{0}: \underline{\underline{e}}_{0}(\underline{w})$. Finally, note that we typically have

$$
\mathcal{L} \subset \mathcal{L}_{\ell}=\left\{q \in L^{2}(\Omega) \mid \int_{\Omega} q \mathrm{~d} \Omega=0\right\} \quad \text { and } \quad x \subset x_{\ell}=H^{1}\left(\Omega_{0}\right)^{3} .
$$

Assumption 3.2. Assume that $s \equiv 0$ and $r \equiv 0$, then $(\underline{\tilde{y}}, \tilde{\mathrm{p}})=(\underline{0}, 0)$ is the unique solution of Eq. (3.3).

One of the aims of this work is to demonstrate that the shear wave generated by the ARF is solution of Eq. (3.3), with a source term $s(t, \underline{w})$ that depends on viscous effects and tissue nonlinearities. The presented analysis is formal, a complete analysis would require to turn most of the assumptions we made into mathematical results and to specify to which functional spaces the various quantities introduced before belong. However, this is a difficult task, since it involves the analysis of the nonlinear elastodynamic problem, that we did not want to address.

\section{Statement of the main results}

Theorem 4.1. Assume that all the hypotheses mentioned above hold. Then,

- the leading-order term $\underline{y}_{0}$ is independent of $\tau$ and the couple $\left(\underline{y}_{0}, \mathrm{p}_{0}\right)$ satisfies Eq. (3.2);

- the first-order corrector term $\underline{y}_{1}$ vanishes;

- the second-order corrector $\underline{y}_{2}$ can be decomposed into two terms $\underline{y}_{2}(\underline{\xi}, t, \tau)=\underline{y}_{p}(\underline{\xi}, t, \tau)+\underline{y}_{s}(\underline{\xi}, t)$, where 
- $\underline{y}_{p}$ is a smooth fast-oscillating, irrotational (pressure) wave and is solution of the mixed problem (in the deformed configuration)

$$
\left\{\begin{array}{lll}
\hat{\rho}_{0} \partial_{\tau}^{2} \underline{y}_{p}-\left(1+\hat{\zeta} \partial_{\tau}\right) \underline{\nabla}_{\underline{x}} p=0 & \text { in } & \Omega(t), \\
p=\operatorname{div}_{\underline{x}} \underline{y}_{p} & \text { in } & \Omega(t), \\
\left(1+\hat{\zeta} \partial_{\tau}\right) p=g & \text { on } & \Gamma(t)
\end{array}\right.
$$

- $\underline{y}_{s}$ is a slowly varying component with prescribed divergence, corresponding to the nonlinear contribution associated with the ARF; the couple $\left(\underline{y}_{s}, \mathrm{p}\right)$ satisfies Eq. (3.3), with $s(t, \underline{w})$ and $r(t, q)$ given by

$$
s(t, \underline{w})=-\int_{\Omega(t)} \underline{w} \cdot \underline{\operatorname{div}}_{\underline{x}} \underline{\underline{\sigma}} A R F
$$

where the symmetric tensors $\underline{\underline{\sigma}}_{A R F}$ and $\underline{\underline{\sigma}}_{b n d y}$ are given by

$$
\underline{\underline{\sigma}}_{A R F}=\frac{\hat{\omega}}{2 \pi} \int_{0}^{\frac{2 \pi}{\hat{\omega}}} \operatorname{div}_{\underline{x}}\left(\left(1+\hat{\zeta} \partial_{\tau}\right) \underline{y}_{p}\right) \underline{\underline{\nabla}}_{\underline{x}} \underline{y}_{p} \mathrm{~d} \tau, \quad \underline{\underline{\sigma}}_{b n d y}=\underline{\underline{\mathbb{1}}} \frac{\hat{\omega}}{2 \pi} \int_{0}^{\frac{2 \pi}{\hat{\omega}}} p^{2} \mathrm{~d} \tau .
$$

Note that the field $\underline{y}_{p}$, solution of (4.1), satisfies at each time $t \in[0, T]$ a periodic problem in $\tau$. For the sake of simplicity, we will now assume that the source term of Problem (4.1) is a combination of a cosine and a sine at a single frequency. As a consequence, the field $\underline{y}_{p}$ can be computed from the solution of a frequency problem. Furthermore, since the field $\underline{y}_{p}$ is irrotational, its computation can be reduced to solving a scalar problem, namely a Helmholtz problem. As shown in the following corollary, these observations can be used to provide a better interpretation of the source term accounting for the ARF phenomenon.

Corollary 4.2. Let the source term of Problem (4.1) be given by

$$
g(\underline{\xi}, t, \tau)=\hat{p}_{c}(\underline{\xi}, t) \cos (\hat{\omega} \tau)+\hat{p}_{s}(\underline{\xi}, t) \sin (\hat{\omega} \tau) .
$$

Then, the scalar field $p$ solution of (4.1) satisfies

$$
p=\operatorname{Re}\left(e^{-i \hat{\omega} \tau} \hat{p}\right)
$$

where $\hat{p}$ is the smooth solution at each time $t$ of the Helmholtz equation

$$
\begin{cases}-\hat{\omega}^{2} \hat{\rho}_{0} \hat{p}-(1-i \hat{\omega} \hat{\zeta}) \Delta_{\underline{x}} \hat{p}=0 & \text { in } \quad \Omega(t) \\ (1-i \hat{\omega} \hat{\zeta}) \hat{p}=\hat{p}_{c}+i \hat{p}_{s} & \text { on } \quad \Gamma(t)\end{cases}
$$


Moreover, there exists another scalar field $\tilde{\mathrm{p}}$ such that the couple $\left(\underline{y}_{s}, \tilde{\mathrm{p}}\right)$ satisfies Eq. (3.3) with

$$
s(t, \underline{w})=\int_{\Omega(t)} \underline{F}_{A R F} \cdot \underline{w} \mathrm{~d} \Omega+\int_{\Gamma_{N}(t)} \underline{w} \cdot \underline{\tilde{\sigma}}_{b n d y} \cdot \underline{n} \mathrm{~d} \Gamma, \quad r(t, q)=\int_{\Omega_{0}} \mathrm{p}_{0} q \mathrm{~d} \Omega_{0},
$$

where $\underline{F}_{A R F}$ is defined as

$$
\underline{F}_{A R F}=-\frac{\hat{\zeta} \hat{\omega}}{2} \operatorname{Im}\left(\hat{p} \underline{\nabla}_{\underline{x}} \overline{\hat{p}}\right)
$$

and $\underline{\underline{\tilde{\sigma}}}_{b n d y}$ is given by

$$
\underline{\underline{\sigma}}_{b n d y}=\frac{1}{4}\left(\frac{1+\hat{\omega}^{2} \hat{\zeta}^{2}}{\hat{\rho}_{0} \hat{\omega}^{2}}\left|\underline{\nabla}_{\underline{x}} \hat{p}\right|^{2}+|\hat{p}|^{2}\right) \underline{\underline{\mathbb{1}}} .
$$

Description of the source term. Note that $\hat{p}$ corresponds to the pushing pressure associated with the ARF. It is computed as the solution of the Helmholtz equation (4.5) with a time-dependent surface source term. The linear form $s(t, \underline{w})$ - that acts as a source term for the shear wave $\underline{y}_{s}$ - depends quadratically on $\hat{p}$ and is composed of a volume integral - that corresponds to the ARF excitation - and a surface term. Note also that the pressure term $\mathrm{p}$ in Theorem 4.1 differs from the term $\tilde{\mathrm{p}}$ in Corollary 4.2. Indeed, we show that the definition of $s(t, \underline{w})$ given in Eq. (4.2) only differs from the one given in Eq. (4.6) by a contribution that is of the form

$$
-\int_{\Omega_{0}} \check{\mathrm{p}}_{\underline{C_{0}}}^{-1}: \underline{\underline{e}}_{0}(\underline{w}) \mathrm{d} \Omega_{0},
$$

and therefore $\tilde{\mathrm{p}}=\mathrm{p}+\check{\mathrm{p}}$.

Boundary source term. The term (4.7) appears naturally in the derivation of Corollary 4.2 and it corresponds to a boundary correction term to the main volumic contribution (4.6). In practice, this boundary correction term is minimised by using the ultrasound probes in such a way to create a Gaussian beam whose amplitude at the focal point is much larger than the amplitude of the emitted ultrasonic waves at the boundary. In this context, the formulation that we have obtained can enable - by numerical simulations - to quantitatively compare the boundary correction term and the main volumic contribution. The prestress-free configuration. If we assume that $\underline{y}_{0}=\underline{0}$, then we simply retrieve that $\Omega(t) \equiv \Omega_{0}$ and $\mathrm{p}_{0}=0$, hence $r(t, q)=0$ for all time. The Helmholtz equation (4.5) and the expression (4.6) are unchanged and the divergence-free field $\underline{y}_{s}$ and $\tilde{\mathrm{p}}$ are solutions of a linear elastodynamic problem in an incompressible transversely isotropic medium.

The dissipation-free case. Let us assume that $\hat{\omega}$ is not close to a resonant frequency of the domain $\Omega(t)$. In that case, the pressure $p$ in (4.5) is well-defined and bounded at the limit $\hat{\zeta} \rightarrow 0$. Therefore, passing to the limit in (4.6), one can see that $\underline{F}_{A R F}=0$ if $\hat{\zeta}$ vanishes and we conclude that viscosity is essential for the generation of shear waves from ARF.

Practical computation of the propagating shear wave. From our formulation, the shear wave field generated by the ARF can be computed as a second-order correction to the nonlinear, time-dependent state associated with the heart dynamics, and it is governed by a linear problem on a time-dependent 
domain. As a consequence, a complete computation of this field is potentially very expensive and complex. However, it is important to note that the time scale of the heart deformation is of the order of one second, therefore it is much slower than the time scale of the shear wave propagation (of the order of one to ten milliseconds). In other words, it is possible to consider three time scales, associated with the heart dynamics, the shear wave propagation and the pressure wave propagation, respectively. Therefore, it is possible to consider a quasi-static approach, and assume that the domain is static with respect to the phenomenon of ARF-induced shear wave propagation and, in turn, with respect to the pressure wave propagation, too. This assumption enables to drastically simplify the mathematical analysis of the problem and its algorithmic implementation.

\section{$5 \quad$ Proof of Theorem 4.1 and Corollary 4.2}

In this section, we explain in detail the procedure used to state Theorem 4.1 and Corollary 4.2. As we have already introduced above, our approach is based on the approximation of $y_{\varepsilon}$ by the first terms of the series expansion in Eq. (3.1). Then, by substituting this expression in Eq. (2.17), we obtain a cascade of equations, and by identification of the equations proportional to the same order of $\varepsilon$, we retrieve the desired results. We give and prove a list of propositions - assuming that all the hypotheses of Sections 2 and 3 hold - that state, in particular, the following results:

- the leading term $\underline{y}_{0}$ corresponds to a purely incompressible displacement (i.e. $J_{0}=1$ ) and does not depend on $\tau$. (Proposition 5.1);

- the term $\underline{y}_{1}$ also does not depend on $\tau$ and is divergence-free (Proposition 5.2);

- the leading term $\underline{y}_{0}$ and a scalar field $\mathrm{p}_{0}$ satisfy the incompressible nonlinear mechanical system (3.2) (Proposition 5.3);

- the term $\underline{y}_{1}$ and a scalar field $\mathrm{p}_{1}$ satisfy Eq. (3.3), with zero source terms and null initial conditions, hence $\underline{y}_{1} \stackrel{\underline{0}}{=} \underline{0}$ (Proposition 5.4);

- the term $\underline{y}_{2}$ can be decomposed as $\underline{y}_{2}=\underline{y}_{p}+\underline{y}_{s}$ where $\underline{y}_{p}$ satisfies Eq. (4.1) - it represents the pressure field associated with the ARF - and $\underline{y}_{s}$ does not depend on $\tau$ (Proposition 5.5);

- the term $\underline{y}_{s}$, together with a scalar field p, is solution of Eq. (3.3), with source terms as in Eqs. (4.2) and (4.3), and it represents the shear wave propagation generated by the ARF (Proposition 5.6).

- in a final step we prove Corollary 4.2. We show that the term $\underline{y}_{s}$ and another scalar field $\tilde{\mathrm{p}}$ are solution of Eq. (3.3) with source terms as in Eqs. (4.6) and (4.7),

\subsection{Preliminaries}

Before describing our approach, we detail the decomposition of the strain and stress tensors resulting from the Ansatz (3.1). We also introduce a decomposition that will be used in what follows to split 
each term $\underline{y}_{i}$ of the expansion (3.1) into slow-varying and fast-varying components. Finally we recall how divergence operators written in the deformed configuration are mapped to differential operators in the reference configuration.

Expansion of strain tensors. From Eq. (3.1), we can rewrite the deformation gradient as

$$
\underline{\underline{F}}_{\varepsilon}:=\underline{\underline{\mathbb{1}}}+\underline{\underline{\nabla}} \underline{y}_{\varepsilon}=\underline{\underline{F}}_{0}+\sum_{i=1}^{N} \varepsilon^{i} \underline{\underline{\nabla}} \underline{y}_{i}+o\left(\varepsilon^{N}\right), \quad \text { with } \quad \underline{\underline{F}}_{0}:=\underline{\underline{\mathbb{1}}}+\underline{\underline{\nabla}} \underline{y}_{0} .
$$

Consequently, the Green-Lagrange tensor reads

$$
\underline{\underline{e}}_{\varepsilon}:=\frac{1}{2}\left(\underline{\underline{\nabla}} \underline{y}_{\varepsilon}+\left(\underline{\underline{\nabla}} \underline{y}_{\varepsilon}\right)^{T}+\left(\underline{\underline{\nabla}} \underline{y}_{\varepsilon}\right)^{T} \cdot \underline{\underline{\nabla}} \underline{y}_{\varepsilon}\right)=\underline{\underline{e}}_{0}+\sum_{i=1}^{N} \varepsilon^{i} \underline{\underline{e}}_{0}\left(\underline{y}_{i}\right)+\sum_{\substack{2 \leq i+j \leq N \\ i, j>0}} \varepsilon^{i+j}\left(\underline{\underline{\nabla}} \underline{y}_{i}\right)^{T} \cdot \underline{\underline{\nabla}} \underline{y} j+o\left(\varepsilon^{N}\right),
$$

with $\underline{\underline{e}}_{0}$ a symmetric tensor quadratic in $\underline{y}_{0}$ and, for every vector $\underline{w}, \underline{\underline{e}}_{0}(\underline{w})$ is a symmetric tensor linear in $\underline{y}_{0}$ and quadratic in $\underline{w}$. These tensors are given by

$$
\underline{\underline{e}}_{0}:=\frac{1}{2}\left(\underline{\underline{F}}_{0}^{T} \cdot \underline{\underline{F}}_{0}-\underline{\underline{\mathbb{1}}}\right) \quad \text { and } \quad \underline{\underline{e}}_{0}(\underline{w}):=\frac{1}{2}\left(\underline{\underline{F}}_{0}^{T} \cdot \underline{\underline{\nabla}} \underline{\underline{w}}+(\underline{\underline{\nabla}} \underline{w})^{T} \cdot \underline{\underline{F}}_{0}\right) .
$$

Again, using the expansion (3.1), we obtain for any vector field $\underline{w}$

$$
d_{\underline{\underline{y}}} \underline{\underline{e}} \cdot \underline{w}:=\frac{1}{2}\left(\underline{\underline{F}}_{\varepsilon}^{T} \cdot \underline{\underline{\nabla}} \underline{w}+(\underline{\underline{\nabla}} \underline{w})^{T} \cdot \underline{\underline{F}}_{\varepsilon}\right)=\underline{\underline{e}}_{0}(\underline{w})+\sum_{i=1}^{N} \varepsilon^{i} \underline{\underline{e}}_{i}(\underline{w})+o\left(\varepsilon^{N}\right),
$$

where, for every $i \in\{1,2, \ldots, N\}, \underline{\underline{e}}_{i}(\underline{w})$ are symmetric tensors linear in $\underline{y}_{i}$ and in $\underline{w}$ and they are given by

$$
\underline{\underline{e}}_{i}(\underline{w}):=\frac{1}{2}\left(\left(\underline{\underline{\nabla}} \underline{y}_{i}\right)^{T} \cdot \underline{\underline{\nabla}} \underline{w}+(\underline{\underline{\nabla}} \underline{w})^{T} \cdot \underline{\underline{\nabla}} \underline{y}_{i}\right) .
$$

Analogously, the Cauchy-Green strain tensor and its inverse read

$$
\underline{\underline{C}} \varepsilon:=\underline{\underline{\mathbb{1}}}+2 \underline{\underline{e}}_{\varepsilon}=\underline{\underline{C}}_{0}+\sum_{i=1}^{N} \varepsilon^{i} \underline{\underline{C}}_{i}+o\left(\varepsilon^{N}\right), \quad \underline{\underline{C}}_{\varepsilon}^{-1}=\underline{\underline{C}}_{0}^{-1}+\sum_{i=1}^{N} \varepsilon^{i} \underline{\underline{G}}_{i}+o\left(\varepsilon^{N}\right), \quad \text { with } \quad \underline{\underline{C}}_{0}=\left(\underline{\underline{\mathbb{1}}}+2 \underline{\underline{e}}_{0}\right),
$$

and we do not detail for the moment the expression of $\underline{\underline{C}}_{i}$ and $\underline{\underline{G}}_{i}$ for $i \geq 1$. The invariant $J_{\varepsilon}$ can be expanded as

$$
J_{\varepsilon}=J_{0}+\sum_{i=1}^{N} \varepsilon^{i} J_{i}+o\left(\varepsilon^{N}\right) \quad \text { with } \quad J_{0}=\operatorname{det} \underline{\underline{F}}_{0}=\left(\operatorname{det} \underline{\underline{C}}_{0}\right)^{\frac{1}{2}},
$$

and the expression of $J_{i}$ is not given for the moment (note that they should not be confused with the reduced invariants classically used in mechanics). Finally, we also have

$$
\underline{\mathrm{t}}_{\varepsilon}=g \underline{\mathrm{t}}_{0}+g \sum_{i=0}^{N} \varepsilon^{i} \underline{\mathrm{t}}_{i}+o\left(\varepsilon^{N}\right) \quad \text { with } \quad \underline{\mathrm{t}}_{0}:=J_{0} \underline{\underline{F}}_{0}^{-T} \cdot \underline{n}_{0} .
$$


The terms $\underline{\mathrm{t}}_{i}$ in the expansion will be given later.

Expansion of stress tensors. The second Piola-Kirchhoff stress tensor accounting for transverse isotropy, near-incompressibility and viscosity can be rewritten in terms of a truncated power series expansion. From Eq. (2.10) and Eq. (3.1) we obtain

$$
\underline{\underline{\Sigma}}_{\varepsilon}^{\mathrm{NI}}:=\frac{1}{2}\left(J_{\varepsilon}^{2}-1\right) \underline{\underline{C}}_{\varepsilon}^{-1}=\sum_{i=0}^{N} \varepsilon^{i} \underline{\underline{\Sigma}}_{i}^{\mathrm{NI}}+o\left(\varepsilon^{N}\right), \quad \text { with } \quad \underline{\underline{\Sigma}}_{0}^{\mathrm{NI}}=\frac{1}{2}\left(J_{0}^{2}-1\right) \underline{\underline{C}}_{0}^{-1} .
$$

In a similar way, we derive the truncated power series expansion in $\varepsilon$ of $\underline{\underline{\Sigma}}_{\varepsilon}^{\mathrm{TI}}$, that reads

$$
\underline{\underline{\Sigma}}_{\varepsilon}^{\mathrm{TI}}=\underline{\underline{\Sigma}}_{0}^{\mathrm{TI}}+\sum_{i=1}^{N} \varepsilon^{i} \underline{\underline{\Sigma}}_{i}^{\mathrm{TI}}+o\left(\varepsilon^{N}\right)
$$

where $\underline{\underline{\Sigma}}_{0}^{\mathrm{TI}}$ is given in Appendix B. The expansion of the second Piola-Kirchhoff stress tensor $\underline{\underline{\Sigma}}_{\varepsilon}^{\mathrm{VS}}$ related to viscosity gives

$$
\underline{\underline{\Sigma}}_{\varepsilon}^{\mathrm{V}}:=\operatorname{tr}\left(\underline{\underline{C}}_{\varepsilon}^{-1} \cdot \underline{\underline{\dot{e}}}_{\varepsilon}\right) \underline{\underline{C}}_{\varepsilon}^{-1}=\sum_{i=-1}^{N-1} \varepsilon^{i} \underline{\underline{\Sigma}}_{i}^{\mathrm{V}}+o\left(\varepsilon^{N-1}\right), \quad \text { with } \quad \underline{\underline{\Sigma}}_{-1}^{\mathrm{V}}=\underline{\underline{C}}_{0}^{-1} \operatorname{tr}\left(\underline{\underline{C}}_{0}^{-1} \cdot \partial_{\tau} \underline{\underline{e}}_{0}\right) .
$$

Note that the series above behaves in $O\left(\varepsilon^{-1}\right)$, due to the fact that differentiation with respect to the fast time variable $\tau$ introduces a factor $\varepsilon^{-1}$ in the expression. Finally, the stress stress tensor $\underline{\underline{\Sigma}}^{A}$ given by (2.11) is independent of $\varepsilon$.

The full expressions of the tensors $\underline{\underline{\Sigma}}_{i}^{\mathrm{NI}}, \underline{\underline{\Sigma}}_{i}^{\mathrm{TI}}$ and $\underline{\underline{\Sigma}}_{i}^{\mathrm{VS}}$ are not detailed here, for the sake of compactness. In fact, we will show that their expression can be considerably simplified, due to the properties of the first terms in the expansion (3.1). More precisely, we will show that $\partial_{\tau} y_{0}=\underline{0}, J_{0}=1$ and $\underline{y}_{1}=\underline{0}$.

Decomposition into fast- and slow-varying terms We decompose $\underline{y}_{i}(\underline{\xi}, t, \tau)$, for $i \in\{0,1, \ldots, N\}$ into two components, namely

$$
\underline{y}_{i}(\underline{\xi}, t, \tau)=\underline{y}_{i}^{f}(\underline{\xi}, t, \tau)+\underline{y}_{i}^{s}(\underline{\xi}, t), \quad \text { with } \quad \underline{y}_{i}^{s}(\underline{\xi}, t)=\frac{\hat{\omega}}{2 \pi} \int_{0}^{\frac{2 \pi}{\hat{\omega}}} \underline{y}_{i}(\underline{\xi}, t, \tau) \mathrm{d} \tau .
$$

The superscript $f$ refers to fast-varying components (with zero average in $\tau$ over its period) whereas $s$ stands for slow-varying terms.

Divergence operators. We give below the relations between divergence operators in the deformed configuration and differential operators in the reference configuration. Starting from the well-known relations

$$
\left(\underline{\nabla}_{\underline{x}} v\right) \circ \underline{\phi}_{0}=\underline{\underline{F}}_{0}^{-T} \cdot \underline{\nabla}\left(v \circ \underline{\phi}_{0}\right), \quad\left(\underline{\underline{\nabla}}_{\underline{x}} \underline{w}\right) \circ \underline{\phi}_{0}=\underline{\underline{\nabla}}\left(\underline{w} \circ \underline{\phi}_{0}\right) \cdot \underline{\underline{F}}_{0}^{-1},
$$


one can show that the divergence of a vector field in the deformed configuration satisfies

$$
\begin{aligned}
\operatorname{div}_{\underline{x}}(\underline{w}) \circ \underline{\phi}_{0}=\operatorname{tr}\left(\left(\underline{\underline{\nabla}}_{\underline{x}} \underline{w}\right) \circ \underline{\phi}_{0}\right) & =\operatorname{tr}\left(\underline{\underline{\nabla}}\left(\underline{w} \circ \underline{\phi}_{0}\right) \cdot \underline{\underline{F}}_{0}^{-1}\right) \\
& =\operatorname{tr}\left(\underline{\underline{F}}_{0}^{-1} \cdot \underline{\underline{\nabla}}\left(\underline{w} \circ \underline{\phi}_{0}\right)\right) \\
& =\operatorname{tr}\left(\underline{\underline{C}}_{0}^{-1} \cdot \underline{\underline{e}}_{0}\left(\underline{w} \circ \underline{\phi}_{0}\right)\right) .
\end{aligned}
$$

Moreover, from all smooth vector fields $\underline{z}$ of $\Omega(t)$ vanishing on the boundary $\Gamma$ we have

$$
\begin{aligned}
\int_{\Omega(t)} \underline{\operatorname{div}}_{\underline{x}}\left(v\left(\underline{\underline{\nabla}}_{\underline{x}} \underline{w}\right)^{T}\right) \cdot \underline{z} \mathrm{~d} \Omega & =-\int_{\Omega(t)} v\left(\underline{\underline{\nabla}}_{\underline{x}} \underline{w}\right)^{T}: \underline{\underline{\nabla}}_{\underline{x}} \underline{\mathrm{d}} \Omega \\
& =-\int_{\Omega_{0}} J_{0}\left(v \circ \phi_{0}\right) \underline{\underline{F}}_{0}^{-T} \cdot\left(\underline{\underline{\nabla}}\left(\underline{w} \circ \underline{\phi}_{0}\right)\right)^{T}: \underline{\underline{\nabla}}\left(\underline{z} \circ \underline{\phi}_{0}\right) \cdot \underline{\underline{F}}_{0}^{-1} \mathrm{~d} \Omega_{0} \\
& =\int_{\Omega_{0}} \underline{\operatorname{div}}\left(J_{0}\left(v \circ \phi_{0}\right) \underline{\underline{F}}_{0}^{-T} \cdot\left(\underline{\underline{\nabla}}\left(\underline{w} \circ \underline{\phi}_{0}\right)\right)^{T} \cdot \underline{\underline{F}}_{0}^{-T}\right)\left(\underline{z} \circ \underline{\phi}_{0}\right) \mathrm{d} \Omega_{0} .
\end{aligned}
$$

Hence, we have shown that

$$
\underline{\operatorname{div}}_{\underline{x}}\left(v\left(\underline{\nabla}_{\underline{x}} \underline{w}\right)^{T}\right) \circ \underline{\phi}_{0}=J_{0}^{-1} \underline{\operatorname{div}}\left(J_{0}\left(v \circ \phi_{0}\right) \underline{\underline{F}}_{0}^{-T} \cdot\left(\underline{\underline{\nabla}}\left(\underline{w} \circ \underline{\phi}_{0}\right)\right)^{T} \cdot \underline{F}_{0}^{-T}\right) .
$$

Relations (5.5) and (5.6) will be used implicitly several times through the proof of the main results.

\subsection{Properties of the leading-order terms}

In Lemma 2.1 we provided a uniform energy estimate. Such estimate can be used rather straightforwardly with the expansion of Eq. (3.1) to deduce some properties of the leading-order terms of the expansion.

Proposition 5.1. The fields $y_{0}$ and $y_{1}$ satisfy

$$
J_{0}=1, \quad \partial_{\tau} \underline{y}_{0}=0, \quad \operatorname{tr}\left(\underline{\underline{C}}_{0}^{-1} \cdot \partial_{t} \underline{\underline{e}}_{0}\right)=0 \quad \text { and } \quad \operatorname{tr}\left(\underline{\underline{C}}_{0}^{-1} \cdot \partial_{\tau} \underline{\underline{e}}_{0}\left(\underline{y}_{1}\right)\right)=0 .
$$

As a consequence, we have

$$
\underline{\underline{\Sigma}}_{0}^{N I}=0 \quad \text { and } \quad \underline{\underline{\Sigma}}_{-1}^{V}=\underline{\underline{\Sigma}}_{0}^{V}=0
$$

Proof. Note that by assumption we have

$$
J_{\varepsilon} \stackrel{\varepsilon \rightarrow 0}{\longrightarrow} J_{0} \quad \text { and } \quad \varepsilon \partial_{t} \underline{y}_{\varepsilon} \stackrel{\varepsilon \rightarrow 0}{\longrightarrow} \partial_{\tau} \underline{y}_{0},
$$

where the limits hold in a sufficiently strong sense. Therefore, by Fatou's lemma, and thanks to the estimate of Lemma 2.1 one can show that

$$
\int_{\Omega_{0}}\left|\partial_{\tau} \underline{y}_{0}\right|^{2} \mathrm{~d} \Omega_{0} \leq \liminf _{\varepsilon \rightarrow 0} \varepsilon^{2} \int_{\Omega_{0}}\left|\partial_{t} \underline{y}_{\varepsilon}\right|^{2} \mathrm{~d} \Omega_{0}=0
$$


As a consequence, $\partial_{\tau} \underline{y}_{0}=0$. Similarly,

$$
\int_{\Omega_{0}}\left(\left(J_{0}^{2}-1\right)-\log \left(J_{0}^{2}\right)\right) \mathrm{d} \Omega_{0} \leq \lim _{\varepsilon \rightarrow 0} \inf \int_{\Omega_{0}}\left(\left(J_{\varepsilon}^{2}-1\right)-\log \left(J_{\varepsilon}^{2}\right)\right) \mathrm{d} \Omega_{0}=0 .
$$

Hence, $J_{0}=1$ and, consequently, the leading term $\underline{y}_{0}$ corresponds to an incompressible deformation. Furthermore, from Jacobi's formula, we obtain

$$
J_{0}^{2}=\operatorname{det} \underline{\underline{C}}_{0}=1 \Longrightarrow 0=\partial_{t} J_{0}^{2}=\operatorname{tr}\left(\underline{\underline{C}}_{0}^{-1} \cdot \partial_{t} \underline{\underline{C}}_{0}\right)=2 \operatorname{tr}\left(\underline{\underline{C}}_{0}^{-1} \cdot \partial_{t} \underline{\underline{e}}_{0}\right)
$$

Now observe that, thanks to definition of $\underline{\underline{e}}_{\varepsilon}$, we retrieve

$$
\begin{aligned}
\operatorname{tr}\left(\underline{\underline{C}}_{\varepsilon}^{-1} \cdot \underline{\underline{e}}_{\varepsilon}\right) & =\varepsilon^{-1} \operatorname{tr}\left(\underline{\underline{C}}_{0}^{-1} \cdot \partial_{\tau} \underline{\underline{e}}_{0}\right)+\operatorname{tr}\left(\underline{\underline{C}}_{0}^{-1} \cdot \partial_{t} \underline{\underline{e}}_{0}\right)+\operatorname{tr}\left(\underline{\underline{C}}_{0}^{-1} \cdot \partial_{\tau} \underline{\underline{e}}_{0}\left(\underline{y}_{1}\right)\right)+O(\varepsilon) \\
& =\operatorname{tr}\left(\underline{\underline{C}}_{0}^{-1} \cdot \partial_{\tau} \underline{\underline{e}}_{0}\left(\underline{y}_{1}\right)\right)+O(\varepsilon) .
\end{aligned}
$$

Therefore - proceeding as before - one can use the energy relation of Lemma 2.1 to show that

$$
\int_{0}^{T} \int_{\Omega_{0}} \operatorname{tr}\left(\underline{\underline{C}}_{0}^{-1} \cdot \partial_{\tau} \underline{\underline{e}}_{0}\left(\underline{y}_{1}\right)\right) \mathrm{d} \Omega_{0} \mathrm{~d} s \leq \lim _{\varepsilon \rightarrow 0} \inf \int_{0}^{T} \int_{\Omega_{0}}\left|\operatorname{tr}\left(\underline{\underline{C}}_{\varepsilon}^{-1} \cdot \underline{\underline{e}}_{\varepsilon}\right)\right|^{2} \mathrm{~d} \Omega_{0} \mathrm{~d} s=0 .
$$

Finally, using Eq. (5.2) one recovers $\underline{\underline{\Sigma}}_{0}^{\mathrm{NI}}=0$, using Eq. (5.3) one gets $\underline{\underline{\Sigma}}_{-1}^{\mathrm{V}}=0$ and using Eq. (5.9) one can deduce that $\underline{\underline{\Sigma}}_{0}^{\mathrm{V}}=0$. As a consequence, one can retrieve (5.7).

\subsection{Properties of the first-order corrector}

We consider the equation associated with $\varepsilon^{-1}$ in Problem (2.17). After some simplifications due to the properties given in Proposition 5.1, one gets

$$
\int_{\Omega_{0}} \rho_{0} \partial_{\tau}^{2} \underline{y}_{1} \cdot \underline{w} \mathrm{~d} \Omega_{0}+\int_{\Omega_{0}} \underline{\underline{\Sigma}}_{1}^{\mathrm{NI}}: \underline{\underline{e}}_{0}(\underline{w}) \mathrm{d} \Omega_{0}=0
$$

Proposition 5.2. The first-order corrector $\underline{y}_{1}$ satisfies

$$
\partial_{\tau} \underline{y}_{1}=\underline{0}, \quad J_{1}=\operatorname{tr}\left(\underline{\underline{F}}_{0}^{-1} \cdot \underline{\underline{\nabla}} \underline{y}_{1}\right)=0 \quad \text { and } \quad \underline{\underline{\Sigma}}_{1}^{N I}=0
$$

Proof. We decompose $\underline{y}_{1}$ as in Eq. (5.4), i.e., $\underline{y}_{1}=\underline{y}_{1}^{f}+\underline{y}_{1}^{s}$ where $\underline{y}_{1}^{s}$ is independent of the fast time variable $\tau$. From Proposition 5.1 we get

$$
0=\operatorname{tr}\left(\underline{\underline{C}}_{0}^{-1} \cdot \partial_{\tau} \underline{\underline{e}}_{0}\left(\underline{y}_{1}\right)\right)=\partial_{\tau} \operatorname{tr}\left(\underline{\underline{C}}_{0}^{-1} \cdot \underline{\underline{e}}_{0}\left(\underline{y}_{1}^{f}\right)\right) .
$$

As $\underline{y}_{1}^{f}$ has zero mean in $\tau$, it must satisfy

$$
\operatorname{tr}\left(\underline{\underline{C}}_{0}^{-1} \cdot \underline{\underline{e}}_{0}\left(\underline{y}_{1}^{f}\right)\right)=0 .
$$


On the other hand, one can show that

$$
\underline{\underline{\Sigma}}_{1}^{\mathrm{NI}}:=\frac{1}{2}\left(J_{0}^{2}-1\right) \underline{\underline{G}}_{1}+J_{1} J_{0} \underline{\underline{C}}_{0}^{-1}=J_{1} \underline{\underline{C}}_{0}^{-1} \quad \text { and } \quad J_{1}:=J_{0} \operatorname{tr}\left(\underline{\underline{C}}_{0}^{-1} \cdot \underline{\underline{e}}_{0}\left(\underline{y}_{1}\right)\right)=\operatorname{tr}\left(\underline{\underline{C}}_{0}^{-1} \cdot \underline{\underline{e}}_{0}\left(\underline{y}_{1}\right)\right) .
$$

Then, if we take $\underline{w}=\underline{y}_{1}^{s}$ in Eq. (5.10) and integrate on $\tau$ over its period, due to the periodicity assumption on all the terms $\partial_{\tau} \underline{y}_{i}$ and $\underline{y}_{i}$ with respect to $\tau$ in $[0,2 \pi / \hat{\omega}]$, we obtain

$$
\int_{0}^{\frac{2 \pi}{\hat{\omega}}} \int_{\Omega_{0}} \underline{\underline{\Xi}}_{1}^{\mathrm{NI}}: \underline{\underline{e}}_{0}\left(\underline{y}_{1}^{s}\right) \mathrm{d} \Omega_{0} \mathrm{~d} \tau=\int_{0}^{\frac{2 \pi}{\hat{\omega}}} \int_{\Omega_{0}} \operatorname{tr}\left(\underline{\underline{C}}_{0}^{-1} \cdot \underline{\underline{e}}_{0}\left(\underline{y}_{1}^{s}\right)\right)^{2} \mathrm{~d} \Omega_{0} \mathrm{~d} \tau=0 \Longrightarrow \operatorname{tr}\left(\underline{\underline{C}}_{0}^{-1} \cdot \underline{\underline{e}}_{0}\left(\underline{y}_{1}^{s}\right)\right)=0 .
$$

Therefore, from Eqs. (5.11) and (5.12) and by linearity,

$$
\operatorname{tr}\left(\underline{\underline{C}}_{0}^{-1} \cdot \underline{\underline{e}}_{0}\left(\underline{y}_{1}\right)\right)=0 \Longrightarrow J_{1}=0 \Longrightarrow \operatorname{tr}\left(\underline{\underline{F}}_{0}^{-1} \cdot \underline{\underline{\nabla}} \underline{y}_{1}\right)=0
$$

and, as a consequence, $\underline{\underline{\Sigma}}_{1}^{\mathrm{NI}}=\underline{\underline{0}}$. Finally, from Eq. (5.10) and using the periodicity and regularity of $\underline{y}_{1}$, we can assert

$$
\int_{\Omega_{0}} \partial_{\tau}^{2} \underline{y}_{1} \cdot \underline{w} \mathrm{~d} \Omega_{0}=0 \Longrightarrow \partial_{\tau}^{2} \underline{y}_{1}=0 \Longrightarrow \partial_{\tau} \underline{y}_{1}=0
$$

thus concluding the proof.

\subsection{The governing equation of the leading term: the underlying nonlinear problem}

The next step of our procedure consists in analysing the equation corresponding to $\varepsilon^{0}$ in the expansion of the elastodynamic problem (2.17). Taking into account the results of the propositions above, its expression reads

$$
\begin{aligned}
\hat{\rho}_{0} \int_{\Omega_{0}}\left(\partial_{\tau}^{2} \underline{y}_{2}+\partial_{t}^{2} \underline{y}_{0}\right) \cdot \underline{w} \mathrm{~d} \Omega_{0}+\int_{\Omega_{0}} \underline{\underline{\Sigma}}_{2}^{\mathrm{NI}}: \underline{\underline{e}}_{0}(\underline{w}) \mathrm{d} \Omega_{0} & +\int_{\Omega_{0}}\left(\underline{\underline{\Sigma}}_{0}^{\mathrm{TI}}+\underline{\underline{\Sigma}}^{A}\right): \underline{\underline{e}}_{0}(\underline{w}) \mathrm{d} \Omega_{0} \\
& +\hat{\zeta} \int_{\Omega_{0}} \underline{\underline{\Sigma}}_{1}^{\mathrm{V}}: \underline{\underline{e}}_{0}(\underline{w}) \mathrm{d} \Omega_{0}=\int_{\Gamma_{0}} g \underline{\mathrm{t}}_{0} \cdot \underline{w} \mathrm{~d} \Gamma_{0} .
\end{aligned}
$$

We highlight that $\underline{\underline{\Sigma}}_{0}^{\mathrm{TI}}$ only depends on $\underline{y}_{0}$ by definition (its expression is given in Appendix B). Hence, it is independent of $\tau$. We also have

$$
\underline{\underline{\Sigma}}_{2}^{\mathrm{NI}}=J_{2} \underline{\underline{C}}_{0}^{-1}
$$

where the detailed expression of $J_{2}$ is not needed now and is given later. Since $\partial_{\tau} \underline{y}_{1}=\underline{0}$, we have

$$
\underline{\underline{\Sigma}}_{1}^{\mathrm{V}}=\underline{\underline{C}}_{0}^{-1} \operatorname{tr}\left(\underline{\underline{G}}_{1} \cdot \partial_{t} \underline{\underline{e}}_{0}\right)+\underline{\underline{C}}_{0}^{-1} \operatorname{tr}\left(\underline{\underline{C}}_{0}^{-1} \cdot \partial_{t} \underline{\underline{e}}_{0}\left(\underline{y}_{1}\right)\right)+\underline{\underline{C}}_{0}^{-1} \operatorname{tr}\left(\underline{\underline{C}}_{0}^{-1} \cdot \partial_{\tau} \underline{\underline{e}}_{0}\left(\underline{y}_{2}\right)\right) .
$$

Proposition 5.3. There exists a scalar field $\mathrm{p}_{0}$ such that the couple $\left(\underline{y}_{0}, \mathrm{p}_{0}\right)$ is solution of the nonlinear elastodynamic equation Eq. (3.2). Moreover,

$$
\frac{\hat{\omega} \hat{\kappa}}{2 \pi} \int_{0}^{\frac{2 \pi}{\hat{\omega}}} J_{2} \mathrm{~d} \tau=\mathrm{p}_{0} \quad \text { and } \quad \underline{\underline{\Sigma}}_{1}^{\mathrm{V}}=\operatorname{tr}\left(\underline{\underline{C}}_{0}^{-1} \cdot \partial_{\tau} \underline{\underline{e}}_{0}\left(\underline{y}_{2}\right)\right) \underline{\underline{C}}_{0}^{-1} .
$$


Proof. We first prove the simplified expression for $\underline{\underline{\Sigma}}_{2}^{\mathrm{V} 1}$. More precisely, our aim is to prove

$$
\operatorname{tr}\left(\underline{\underline{G}}_{1} \cdot \partial_{t} \underline{\underline{e}}_{0}\right)+\operatorname{tr}\left(\underline{\underline{C}}_{0}^{-1} \cdot \partial_{t} \underline{\underline{e}}_{0}\left(\underline{y}_{1}\right)\right)=0
$$

First, note that from Eq. (5.13) we have

$$
\partial_{t} \operatorname{tr}\left(\underline{\underline{C}}_{0}^{-1} \cdot \underline{\underline{e}}_{0}\left(\underline{y}_{1}\right)\right)=0 \Longrightarrow \operatorname{tr}\left(\underline{\underline{C}}_{0}^{-1} \cdot \partial_{t} \underline{\underline{e}}_{0}\left(\underline{y}_{1}\right)\right)=-\operatorname{tr}\left(\partial_{t} \underline{\underline{C}}_{0}^{-1} \cdot \underline{\underline{e}}_{0}\left(\underline{y}_{1}\right)\right)
$$

Now, by definition,

$$
\operatorname{tr}\left(\underline{\underline{G}}_{1} \cdot \partial_{t} \underline{\underline{e}}_{0}\right)=\operatorname{tr}\left(-2 \underline{\underline{C}}_{0}^{-1} \cdot \underline{\underline{e}}_{0}\left(\underline{y}_{1}\right) \cdot \underline{\underline{C}}_{0}^{-1} \cdot \partial_{t} \underline{\underline{e}}_{0}\right)=\operatorname{tr}\left(-2 \underline{\underline{C}}_{0}^{-1} \cdot \partial_{t} \underline{\underline{e}}_{0} \cdot \underline{\underline{C}}_{0}^{-1} \cdot \underline{\underline{e}}_{0}\left(\underline{y}_{1}\right)\right) .
$$

Moreover, note that

$$
\partial_{t} \underline{\underline{C}}_{0}^{-1}=-\underline{\underline{C}}_{0}^{-1} \cdot \partial_{t} \underline{\underline{C}}_{0} \cdot \underline{\underline{C}}_{0}^{-1}=-2 \underline{\underline{C}}_{0}^{-1} \cdot \partial_{t} \underline{\underline{e}}_{0} \cdot \underline{\underline{C}}_{0}^{-1}
$$

Therefore, we can state that

$$
\operatorname{tr}\left(\underline{\underline{G}}_{1} \cdot \partial_{t} \underline{\underline{e}}_{0}\right)=\operatorname{tr}\left(\partial_{t} \underline{\underline{C}}_{0}^{-1} \cdot \underline{\underline{e}}_{0}\left(\underline{y}_{1}\right)\right)
$$

Combining Eqs. (5.17) and (5.19), we obtain Eq. (5.16), which concludes the first part of the proof. Finally, in order to derive an expression for the governing equation of $\underline{y}_{0}$, we need to discard the terms depending on the fast time variable $\tau$ in Eq. (5.14). Therefore, we integrate Eq. (5.14) on $\tau$ over its period. Since $\underline{y}_{2}$ is smooth and periodic in $\tau$, and $g$ has zero mean value on the same period, we derive Eq. (3.2) using the expression of the stress tensors, as desired.

Remark Note that the viscous term does not give any contribution at this stage. Furthermore, (5.15) relates higher-order corrector terms to $\mathrm{p}_{0}$. In what follows, we will show that it is a constraint on the divergence of the (slowly varying) second-order corrector $\underline{y}_{2}$.

\subsection{The governing equation of the first-order corrector}

We are now interested in the equation associated with $\varepsilon$ in Problem (2.17). It reads

$$
\begin{aligned}
\hat{\rho}_{0} \int_{\Omega_{0}}\left(\partial_{\tau}^{2} \underline{y}_{3}+2 \partial_{t \tau}^{2} \underline{y}_{2}+\partial_{t}^{2} \underline{y}_{1}\right) \cdot \underline{w} \mathrm{~d} \Omega_{0}+\int_{\Omega_{0}}\left(\left(\underline{\underline{\Sigma}}_{0}^{\mathrm{TI}}+\underline{\underline{\Sigma}}^{A}\right): \underline{\underline{e}}_{1}(\underline{w})+\underline{\underline{\Sigma}}_{1}^{\mathrm{TI}}: \underline{\underline{e}}_{0}(\underline{w})\right) \mathrm{d} \Omega_{0} \\
+\int_{\Omega_{0}}\left(\underline{\underline{\Sigma}}_{2}^{\mathrm{NI}}: \underline{\underline{e}}_{1}(\underline{w})+\underline{\underline{\Sigma}}_{3}^{\mathrm{NI}}: \underline{\underline{e}}_{0}(\underline{w})\right) \mathrm{d} \Omega_{0}+\hat{\zeta} \int_{\Omega_{0}}\left(\underline{\underline{\Sigma}}_{1}^{\mathrm{V}}: \underline{\underline{e}}_{1}(\underline{w})+\underline{\underline{\Sigma}}_{2}^{\mathrm{V}}: \underline{\underline{e}}_{0}(\underline{w})\right) \mathrm{d} \Omega_{0} \\
\quad=\int_{\Gamma_{0}} g \underline{\mathrm{t}}_{1} \cdot \underline{w} \mathrm{~d} \Gamma_{0} .
\end{aligned}
$$

After some algebra, the terms $\underline{\underline{\Sigma}}_{3}^{\mathrm{NI}}$ and $\underline{\Sigma}_{2}^{\mathrm{V}}$ read:

$$
\underline{\underline{\Sigma}}_{3}^{\mathrm{NI}}=J_{2} \underline{\underline{G}}_{1}+J_{3} \underline{\underline{C}}_{0}^{-1} \quad \text { and } \quad \underline{\underline{\Sigma}}_{2}^{\mathrm{V}}=\operatorname{tr}\left(\underline{\underline{C}}_{0}^{-1} \cdot \partial_{\tau} \underline{\underline{e}}_{0}\left(\underline{y}_{2}\right)\right) \underline{\underline{G}}_{1}+\operatorname{tr}\left(\underline{\underline{D}}_{3}\right) \underline{\underline{C}}_{0}^{-1}
$$


where $\underline{\underline{D}}_{3}$ is a tensor depending on $\left(\underline{y}_{0}, \underline{y}_{1}, \underline{y}_{2}, \underline{y}_{3}\right)$, whose expression is not used. The detailed expression of the tensor $\sum_{1}^{\mathrm{TI}}$ can be found in Appendix B. For the sake of completeness, we recall here that it corresponds to

$$
\underline{\underline{\Sigma}}_{1}^{\mathrm{TI}}=\underline{\underline{\mathbf{A}}}_{0}: \underline{\underline{e}}_{0}\left(\underline{y}_{1}\right)
$$

Therefore, it does not depend on $\tau$. Since we want to derive the governing equation of $\underline{y}_{1}$ (that does not depend on $\tau$ as well), it is natural to integrate Eq. (5.20) on $\tau$ over $[0, \hat{\omega} /(2 \pi)]$. Using similar arguments to the previous section (namely, the periodicity of the quantities depending on $\tau$ ), one can show that

$$
\begin{aligned}
\hat{\rho}_{0} \int_{\Omega_{0}} \partial_{t}^{2} \underline{y}_{1} \cdot \underline{w} \mathrm{~d} \Omega_{0}+\int_{\Omega_{0}} \mathrm{p}_{1} \underline{\underline{C}}_{0}^{-1}: \underline{\underline{e}}_{0}(\underline{w}) \mathrm{d} \Omega_{0}+\int_{\Omega_{0}} \underline{\underline{\Sigma}}_{1}^{\mathrm{TI}}: \underline{\underline{e}}_{0}(\underline{w}) \mathrm{d} \Omega_{0}+\int_{\Omega_{0}} \mathrm{p}_{0} \underline{\underline{G}}_{1}: \underline{e}_{0}(\underline{w}) \mathrm{d} \Omega_{0} \\
+\int_{\Omega_{0}}\left(\underline{\underline{\Sigma}}_{0}^{\mathrm{TI}}+\underline{\underline{\Sigma}}^{A}+\mathrm{p}_{0} \underline{\underline{C}}_{0}^{-1}\right): \underline{\underline{e}}_{1}(\underline{w}) \mathrm{d} \Omega_{0}=0
\end{aligned}
$$

where the scalar field $\mathrm{p}_{1}$ satisfies

$$
\frac{\hat{\omega}}{2 \pi} \int_{0}^{\frac{2 \pi}{\hat{\omega}}}\left(J_{3}+\hat{\zeta} \operatorname{tr}\left(\underline{\underline{D}}_{3}\right)\right) \mathrm{d} \tau=\mathrm{p}_{1} .
$$

Proposition 5.4. The first-order corrector $\underline{y}_{1}$ satisfies

$$
\underline{y}_{1}=\underline{0} .
$$

Proof. The proof consists in showing that the couple $\left(\underline{y}_{1}, \mathrm{p}_{1}\right)$ satisfies Problem (3.3) with no source terms. First, by algebraic manipulations and using the definition of the stress tensors, it is easy to see that Eq. (5.21) corresponds to the first equation of Eq. (3.3) with $\underline{\tilde{y}}=\underline{y}, \tilde{p}=\mathrm{p}_{1}$, and $s(t, \underline{w})=0$. Then, since we have shown previously that $J_{1}=0$, we can write

$$
\int_{\Omega_{0}} \underline{\underline{C}}_{0}^{-1}: \underline{\underline{e}}_{0}\left(\underline{y}_{1}\right) q \mathrm{~d} \Omega_{0}=0
$$

retrieving the second equation in (3.3), with $r(t, q)$ equal to zero. Finally, since initial conditions are assumed to be zero, and Problem (3.3) is well-posed by assumption, the only solution is

$$
\underline{y}_{1}=\underline{0}, \quad \mathrm{p}_{1}=0
$$

thus completing the proof.

Remark Since $\underline{y}_{1}$ vanishes, numerous simplifications can be deduced in the expansion of the mechanical quantities in Section 5.1. Among them, one can deduce that

$$
\underline{\underline{C}}_{1}=\underline{\underline{0}}, \quad J_{2}=\operatorname{tr}\left(\underline{\underline{C}}_{0}^{-1} \cdot \underline{\underline{e}}_{0}\left(\underline{y}_{2}\right)\right), \quad \underline{\underline{G}}_{2}=-2 \underline{\underline{C}}_{0}^{-1} \cdot \underline{\underline{e}}_{0}\left(\underline{y}_{2}\right) \cdot \underline{\underline{C}}_{0}^{-1}
$$

and

$$
\underline{\mathrm{t}}_{1}=\underline{0}, \quad \underline{e}_{\varepsilon}=\underline{\underline{e}}_{0}+\varepsilon^{2} \underline{\underline{e}}_{0}\left(\underline{y}_{2}\right)+\varepsilon^{3} \underline{\underline{e}}_{0}\left(\underline{y}_{3}\right)+o\left(\varepsilon^{3}\right) \text {. }
$$




\subsection{The governing equation of the fast-oscillating second-order corrector: the ARF pressure field}

The second main result of this work concerns the derivation of the governing equation of the pressure wave propagation associated with the ARF. To do so, we consider again the term corresponding to $\varepsilon^{0}$ in the expansion of Problem (2.17). More precisely, if we substitute Eq. (3.2) in Eq. (5.14), we obtain

$$
\hat{\rho}_{0} \int_{\Omega_{0}} \partial_{\tau}^{2} \underline{y}_{2} \cdot \underline{w} \mathrm{~d} \Omega_{0}+\int_{\Omega_{0}}\left(\underline{\underline{\Sigma}}_{2}^{\mathrm{NI}}-\mathrm{p}_{0} \underline{\underline{C}}_{0}^{-1}\right): \underline{\underline{e}}_{0}(\underline{w}) \mathrm{d} \Omega_{0}+\hat{\zeta} \int_{\Omega_{0}} \underline{\underline{\Sigma}}_{1}^{\mathrm{V}}: \underline{\underline{e}}_{0}(\underline{w}) \mathrm{d} \Omega_{0}=\int_{\Gamma_{0}} g \underline{\mathrm{t}}_{0} \cdot \underline{w} \mathrm{~d} \Gamma_{0}
$$

where $\underline{\underline{V}}_{1}^{\mathrm{V}}$ is given in Eq. (5.15) and

$$
\underline{\underline{\Sigma}}_{2}^{\mathrm{NI}}-\mathrm{p}_{0} \underline{\underline{C}}_{0}^{-1}=\left(J_{2}-\frac{\hat{\omega}}{2 \pi} \int_{0}^{\frac{2 \pi}{\hat{\omega}}} J_{2} \mathrm{~d} \tau\right) \underline{\underline{C}}_{0}^{-1}
$$

Now, to go further in our analysis, we need to decompose $\underline{y}_{2}$ as in Eq. (5.4) into a fast and a slowly varying term. We define

$$
\underline{y}_{2}=\underline{y}_{p}+\underline{y}_{s} \quad \text { with } \quad \underline{y}_{p}:=\underline{y}_{2}^{f} \quad \text { and } \quad \underline{y}_{s}:=\underline{y}_{2}^{s} .
$$

From Eq. (5.23) and Eq. (5.24) one can deduce that

$$
\underline{\underline{\Sigma}}_{2}^{\mathrm{NI}}-\mathrm{p}_{0} \underline{\underline{C}}_{0}^{-1}=\operatorname{tr}\left(\underline{\underline{C}}_{0}^{-1} \cdot \underline{\underline{e}}_{0}\left(\underline{y}_{p}\right)\right) \underline{\underline{C}}_{0}^{-1} \quad \text { hence } \quad \underline{\underline{\Sigma}}_{1}^{\mathrm{V}}=\partial_{\tau}\left(\underline{\underline{\Sigma}}_{2}^{\mathrm{NI}}-\mathrm{p}_{0} \underline{\underline{C}}_{0}^{-1}\right) .
$$

The main result of this section is the following proposition.

Proposition 5.5. There exist a scalar field $p$ such that $\left(\underline{y}_{p}, p\right)$ satisfies Eq. (4.1).

Proof. Let us consider Eq. (5.25). If we use Eq. (5.28) and the definition of the stress tensor $\underline{\underline{\Sigma}}_{1}^{\mathrm{V}}$, we obtain

$$
\begin{aligned}
\hat{\rho}_{0} \int_{\Omega_{0}} \partial_{\tau}^{2} \underline{y}_{p} \cdot \underline{w} \mathrm{~d} \Omega_{0}+ & \int_{\Omega_{0}} \operatorname{tr}\left(\underline{\underline{C}}_{0}^{-1} \cdot \underline{\underline{e}}_{0}\left(\underline{y}_{p}\right)\right) \operatorname{tr}\left(\underline{\underline{C}}_{0}^{-1} \cdot \underline{\underline{e}}_{0}(\underline{w})\right) \mathrm{d} \Omega_{0} \\
& +\hat{\zeta} \int_{\Omega_{0}} \operatorname{tr}\left(\underline{\underline{C}}_{0}^{-1} \cdot \partial_{\tau} \underline{\underline{e}}_{0}\left(\underline{y}_{p}\right)\right) \operatorname{tr}\left(\underline{\underline{C}}_{0}^{-1} \cdot \underline{\underline{e}}_{0}(\underline{w})\right) \mathrm{d} \Omega_{0}=\int_{\Gamma_{0}} g \underline{w} \cdot \underline{\underline{F}}_{0}^{-T} \cdot \underline{n}_{0} \mathrm{~d} \Gamma_{0} .
\end{aligned}
$$

Since $J_{0}=1$, the equation above can be rewritten in the deformed configuration as

$$
\hat{\rho}_{0} \int_{\Omega(t)} \partial_{\tau}^{2} \underline{y}_{p} \cdot \underline{w} \mathrm{~d} \Omega+\int_{\Omega(t)} \operatorname{div}_{\underline{x}} \underline{y}_{p} \cdot \operatorname{div}_{\underline{x}} \underline{w} \mathrm{~d} \Omega+\hat{\zeta} \int_{\Omega(t)} \operatorname{div}_{\underline{x}} \partial_{\tau} \underline{y}_{p} \cdot \operatorname{div}_{\underline{x}} \underline{w} \mathrm{~d} \Omega=\int_{\Gamma(t)} g \underline{n} \cdot \underline{w} \mathrm{~d} \Gamma .
$$


In strong form, Eq. (5.30) reads

$$
\begin{cases}\hat{\rho}_{0} \partial_{\tau}^{2} \underline{y}_{p}-\underline{\nabla}_{\underline{x}} \operatorname{div}_{\underline{x}} \underline{y}_{p}-\hat{\zeta} \underline{\nabla}_{\underline{x}} \operatorname{div}_{\underline{x}} \partial_{\tau} \underline{y}_{p}=0 & \text { in } \quad \Omega(t), \\ \left(1+\hat{\zeta} \partial_{\tau}\right) \operatorname{div}_{\underline{x}} \underline{y}_{p} \cdot \underline{n}=g \underline{n} & \text { on } \quad \Gamma(t) .\end{cases}
$$

Finally, observe that Eq. (5.31) can be recast as the mixed problem (4.1).

Remark Eq. (5.31) shows that $\underline{y}_{p}$ is irrotational: indeed, since $\hat{\rho}_{0}$ and $\hat{\zeta}$ are constant, we have $\underline{\nabla}_{\underline{x}} \times \underline{y}_{p}=0$.

From Eq. (5.29) it is possible to derive an expression of the boundary condition satisfied by $\underline{y}_{p}$ in the Lagrangian framework. More precisely, one can use the following property

$$
\begin{aligned}
\int_{\Omega_{0}} \operatorname{tr}\left(\underline{\underline{C}}_{0}^{-1} \cdot \underline{\underline{e}}_{0}\left(\underline{y}_{p}\right)\right) \operatorname{tr}\left(\underline{\underline{C}}_{0}^{-1} \cdot \underline{\underline{e}}_{0}(\underline{w})\right) \mathrm{d} \Omega_{0}=\int_{\Omega_{0}} \operatorname{tr}\left(\underline{\underline{C}}_{0}^{-1} \cdot \underline{\underline{e}}_{0}\left(\underline{y}_{p}\right)\right) \underline{\underline{F}}_{0}^{-T}: \underline{\underline{\nabla}} \underline{\underline{w}} \mathrm{~d} \Omega_{0} \\
=-\int_{\Omega_{0}} \underline{\operatorname{div}}\left(\operatorname{tr}\left(\underline{\underline{C}}_{0}^{-1} \cdot \underline{\underline{e}}_{0}\left(\underline{y}_{p}\right)\right) \underline{\underline{F}}_{0}^{-T}\right) \cdot \underline{w} \mathrm{~d} \Omega_{0}+\int_{\Gamma_{0}} \operatorname{tr}\left(\underline{\underline{C}}_{0}^{-1} \cdot \underline{\underline{e}}_{0}\left(\underline{y}_{p}\right)\right) \underline{w} \cdot \underline{\underline{F}}_{0}^{-T} \cdot \underline{n} \mathrm{~d} \Gamma_{0},
\end{aligned}
$$

to deduce that

$$
\left(1+\hat{\zeta} \partial_{\tau}\right) \operatorname{tr}\left(\underline{\underline{C}}_{0}^{-1} \cdot \underline{\underline{e}}_{0}\left(\underline{y}_{p}\right)\right)=g \quad \text { on } \quad \Gamma_{0}
$$

Note that the equality above could be also deduced from (5.31) after composition with $\underline{\phi}_{0}^{-1}$.

\subsection{The governing equation of the slow-varying second-order corrector: the shear wave propagation}

At last, we give an explicit expression of the governing equation for the second-order corrector $\underline{y}_{s}=\underline{y}_{2}^{s}$ as defined in Eq. (5.27). To this end, we need to evaluate the term corresponding to $\varepsilon^{2}$ in the expansion of Problem (2.17). Using the results above, namely, $J_{0}=1, \partial_{\tau} \underline{y}_{0}=\underline{0}, \underline{y}_{1}=\underline{0}$, it reads

$$
\begin{aligned}
& \hat{\rho}_{0} \int_{\Omega_{0}}\left(\partial_{\tau}^{2} \underline{y}_{4}+2 \partial_{t \tau}^{2} \underline{y}_{3}+\partial_{t}^{2} \underline{y}_{2}\right) \cdot \underline{w} \mathrm{~d} \Omega_{0}+\int_{\Omega_{0}}\left(\underline{\underline{\Sigma}}_{4}^{\mathrm{NI}}: \underline{\underline{e}}_{0}(\underline{w})+\underline{\underline{\Sigma}}_{2}^{\mathrm{NI}}: \underline{\underline{e}}_{2}(\underline{w})\right) \mathrm{d} \Omega_{0} \\
&+\int_{\Omega_{0}}\left(\underline{\underline{\Sigma}}_{2}^{\mathrm{TI}}: \underline{\underline{e}}_{0}(\underline{w})+\left(\underline{\underline{\Sigma}}_{0}^{\mathrm{TI}}+\underline{\underline{\Sigma}}^{A}\right): \underline{\underline{e}}_{2}(\underline{w})\right) \mathrm{d} \Omega_{0} \\
& \quad+\hat{\zeta} \int_{\Omega_{0}}\left(\underline{\underline{\Sigma}}_{3}^{\mathrm{V}}: \underline{\underline{e}}_{0}(\underline{w})+\underline{\underline{\Sigma}}_{1}^{\mathrm{V}}: \underline{\underline{e}}_{2}(\underline{w})\right) \mathrm{d} \Omega_{0}=\int_{\Gamma_{0}} g \underline{\mathrm{t}}_{2} \cdot \underline{w} \mathrm{~d} \Gamma_{0} .
\end{aligned}
$$

Note that, since $\underline{y}_{1}=\underline{0}$, the equation above only involves even terms of the expansion of the stress tensors. Moreover, we have $\underline{\underline{\Sigma}}_{2}^{\mathrm{TI}}=\underline{\underline{\mathbf{A}}}_{0}: \underline{\underline{e}}_{0}\left(\underline{y}_{2}\right)$ and $\underline{\underline{\mathbf{A}}}_{0}$ is given in Appendix B. We just highlight here that it is linear in $\underline{y}_{2}$. We recall that

$$
\underline{\underline{\Sigma}}_{2}^{\mathrm{NI}}=\operatorname{tr}\left(\underline{\underline{C}}_{0}^{-1} \cdot \underline{\underline{e}}_{0}\left(\underline{y}_{2}\right)\right) \underline{\underline{C}}_{0}^{-1}=J_{2} \underline{\underline{C}}_{0}^{-1}, \quad \underline{\underline{\Sigma}}_{1}^{\mathrm{V}}=\operatorname{tr}\left(\underline{\underline{C}}_{0}^{-1} \cdot \partial_{\tau} \underline{\underline{e}}_{0}\left(\underline{y}_{2}\right)\right) \underline{\underline{C}}_{0}^{-1}=\left(\partial_{\tau} J_{2}\right) \underline{\underline{C}}_{0}^{-1}=\partial_{\tau} \underline{\underline{\Sigma}}_{2}^{\mathrm{NI}} .
$$


Finally, since $\underline{y}_{1}=0$, the terms $\underline{\underline{\Sigma}}_{4}^{\mathrm{NI}}$ and $\underline{\underline{\Sigma}}_{3}^{\mathrm{V}}$ reduce to

$$
\underline{\underline{\Sigma}}_{4}^{\mathrm{NI}}=J_{2} \underline{\underline{G}}_{2}+\left(J_{2}^{2} / 2+J_{4}\right) \underline{\underline{C}}_{0}^{-1}, \quad \underline{\underline{\Sigma}}_{3}^{\mathrm{V}}=\left(\partial_{\tau} J_{2}\right) \underline{\underline{G}}_{2}+\operatorname{tr}\left(\underline{\underline{D}}_{4}\right) \underline{\underline{C}}_{0}^{-1},
$$

where $\underline{\underline{D}}_{4}$ is a tensor field and $J_{4}$ a scalar field whose exact expressions are not needed, and where the expression of $\underline{\underline{G}}_{2}=-2 \underline{\underline{C}}_{0}^{-1} \cdot \underline{\underline{e}}_{0}\left(\underline{y}_{2}\right) \cdot \underline{\underline{C}}_{0}^{-1}$ has already been given in Eq. 5.23. We want to derive the governing equation of the slowly varying vector field $\underline{y}_{2}^{S}$ given in the decomposition Eq. (5.27), therefore we integrate Eq. (5.34) on $\tau$ over its period. Since this last step is fundamental, we now detail each term. First, we use the decomposition $\underline{y}_{2}=\underline{y}_{p}+\underline{y}_{s}$ to write

$$
\hat{\rho}_{0} \frac{\hat{\omega}}{2 \pi} \int_{0}^{\frac{2 \pi}{\hat{\omega}}}\left(\partial_{\tau}^{2} \underline{y}_{4}+2 \partial_{t \tau}^{2} \underline{y}_{3}+\partial_{t}^{2} \underline{y}_{2}\right) \cdot \underline{w} \mathrm{~d} \tau=\hat{\rho}_{0} \partial_{t}^{2} \underline{y}_{s} .
$$

In addition, it is possible to show that

$$
\begin{aligned}
\frac{\hat{\omega}}{2 \pi} \int_{0}^{\frac{2 \pi}{\omega}}\left(\underline{\underline{\Sigma}}_{2}^{\mathrm{NI}}+\hat{\zeta} \underline{\underline{\Sigma}}_{1}^{\mathrm{V}}\right): \underline{\underline{e}}_{2}(\underline{w}) \mathrm{d} \tau=\mathrm{p}_{0} \underline{\underline{C}}_{0}^{-1}: \underline{\underline{e}}_{s}(\underline{w}) \\
\\
\quad+\frac{\hat{\omega}}{2 \pi} \int_{0}^{\frac{2 \pi}{\hat{\omega}}}\left(1+\hat{\zeta} \partial_{\tau}\right) \operatorname{tr}\left(\underline{\underline{C}}_{0}^{-1} \cdot \underline{e}_{0}\left(\underline{y}_{p}\right)\right) \underline{\underline{C}}_{0}^{-1}: \underline{\underline{e}}_{p}(\underline{w}) \mathrm{d} \tau,
\end{aligned}
$$

where we have defined

$$
\underline{\underline{e}}_{s}(\underline{w}):=\frac{1}{2}\left(\left(\underline{\underline{\nabla}} \underline{\underline{y}}_{s}\right)^{T} \cdot \underline{\underline{\nabla}} \underline{w}+(\underline{\underline{\nabla w}})^{T} \cdot \underline{\underline{\nabla}} \underline{y}_{s}\right), \quad \underline{\underline{e}}_{p}(\underline{w}):=\frac{1}{2}\left(\left(\underline{\underline{\nabla}} \underline{p}_{p}\right)^{T} \cdot \underline{\underline{\nabla}} \underline{\underline{w}}+(\underline{\underline{\nabla w}})^{T} \cdot \underline{\underline{\nabla}} \underline{y}_{p}\right) .
$$

Similarly, one can show that there exists a scalar field p independent of $\tau$ such that

$$
\begin{aligned}
\frac{\hat{\omega}}{2 \pi} \int_{0}^{\frac{2 \pi}{\hat{\omega}}}\left(\underline{\underline{\Sigma}}_{4}^{\mathrm{NI}}+\hat{\zeta} \underline{\underline{\Sigma}}_{3}^{\mathrm{V}}\right): \underline{\underline{e}}_{0}(\underline{w}) \mathrm{d} \tau & =\mathrm{p} \underline{\underline{C}}_{0}^{-1}: \underline{\underline{e}}_{0}(\underline{w}) \\
& -\frac{\hat{\omega}}{\pi} \int_{0}^{\frac{2 \pi}{\hat{\omega}}}\left(1+\hat{\zeta} \partial_{\tau}\right) \operatorname{tr}\left(\underline{\underline{C}}_{0}^{-1} \cdot \underline{e}_{0}\left(\underline{y}_{p}\right)\right) \underline{\underline{C}}_{0}^{-1} \cdot \underline{\underline{e}}_{0}\left(\underline{y}_{p}\right) \cdot \underline{\underline{C}}_{0}^{-1}: \underline{e}_{0}(\underline{w}) \mathrm{d} \tau .
\end{aligned}
$$

Finally, the transversely isotropic tensors and the activation term satisfy

$$
\frac{\hat{\omega}}{2 \pi} \int_{0}^{\frac{2 \pi}{\hat{\omega}}}\left(\underline{\underline{\Sigma}}_{2}^{\mathrm{TI}}: \underline{\underline{e}}_{0}(\underline{w})+\left(\underline{\underline{\Sigma}}_{0}^{\mathrm{TI}}+\underline{\underline{\Sigma}}^{A}\right): \underline{\underline{e}}_{2}(\underline{w})\right) \mathrm{d} \tau=\underline{\underline{e}}_{0}\left(\underline{y}_{s}\right): \underline{\underline{\mathbf{A}}}_{0}: \underline{\underline{e}}_{0}(\underline{w})+\left(\underline{\underline{\Sigma}}_{0}^{\mathrm{TI}}+\underline{\underline{\Sigma}}^{A}\right): \underline{\underline{e}}_{s}(\underline{w}) .
$$

Note that we have introduced the unknown $\mathrm{p}$ and we need an equation for it. In order to obtain this equation, we simply observe that from Eq. (5.15) and the expression of $J_{2}$ given in Eq. 5.23, we have

$$
\mathrm{p}_{0}=\frac{\hat{\omega}}{2 \pi} \int_{0}^{\frac{2 \pi}{\hat{\omega}}} J_{2} \mathrm{~d} \tau=\operatorname{tr}\left(\underline{\underline{C}}_{0}^{-1} \cdot \underline{\underline{e}}_{0}\left(\underline{y}_{s}\right)\right) \text {. }
$$

We can now state the main result of this section. 
Proposition 5.6. There exists a scalar field $\mathrm{p}$ such that the couple $\left(\underline{y}_{s}, \mathrm{p}\right)$ is solution of Eq. (3.3), with

$$
\begin{aligned}
s(t, \underline{w})=-\frac{\hat{\omega}}{2 \pi} \int_{0}^{\frac{2 \pi}{\omega}} \int_{\Omega(t)} \underline{\operatorname{div}}_{\underline{x}}\left(\operatorname{div}_{\underline{x}}\left(\left(1+\hat{\zeta} \partial_{\tau}\right) \underline{y}_{p}\right) \underline{\underline{\nabla}}_{\underline{x}} \underline{y}_{p}\right) \cdot \underline{w} \mathrm{~d} \Omega \mathrm{d} \tau & \\
& +\int_{\Gamma(t)}\left(\frac{\hat{\omega}}{2 \pi} \int_{0}^{\frac{2 \pi}{\omega}} p^{2} \mathrm{~d} \tau\right) \underline{w} \cdot \underline{n} \mathrm{~d} \Gamma,
\end{aligned}
$$

where $p=\operatorname{tr}\left(\underline{\underline{C}}_{0}^{-1} \cdot \underline{\underline{e}}_{0}\left(\underline{y}_{p}\right)\right)$ and

$$
r(t, q)=\int_{\Omega_{0}} \mathrm{p}_{0} q \mathrm{~d} \Omega_{0}
$$

Proof. Considering Eqs. (5.35), (5.36) and (5.37), one can see that the couple $\left(\underline{y}_{s}, \mathrm{p}\right)$ satisfies the first equation in (3.3) with source term $s(t, \underline{w})$ given by

$$
\begin{aligned}
s(t, \underline{w}) & =\frac{\hat{\omega}}{2 \pi} \int_{0}^{\frac{2 \pi}{\hat{\omega}}} \int_{\Gamma_{0}} g \underline{\mathrm{t}}_{2} \cdot \underline{w} \mathrm{~d} \Gamma_{0} \mathrm{~d} \tau \\
& -\frac{\hat{\omega}}{2 \pi} \int_{0}^{\frac{2 \pi}{\hat{\omega}}} \int_{\Omega_{0}}\left(1+\hat{\zeta} \partial_{\tau}\right) \operatorname{tr}\left(\underline{\underline{C}}_{0}^{-1} \cdot \underline{\underline{e}}_{0}\left(\underline{y}_{p}\right)\right)\left(\underline{\underline{C}}_{0}^{-1}: \underline{\underline{e}}_{p}(\underline{w})-2 \underline{\underline{C}}_{0}^{-1} \cdot \underline{\underline{e}}_{0}\left(\underline{y}_{p}\right) \cdot \underline{\underline{C}}_{0}^{-1}: \underline{\underline{e}}_{0}(\underline{w})\right) \mathrm{d} \Omega_{0} \mathrm{~d} \tau .
\end{aligned}
$$

Moreover, from Eq. (5.39) one can see that the second equation in (3.3) is satisfied with $r(t, q)$ given by

$$
r(t, q)=\int_{\Omega_{0}} \mathrm{p}_{0} q \mathrm{~d} \Omega_{0}
$$

In order to conclude the proof, we only need to check that $s(t, \underline{w})$ can be rewritten as in Eq. (5.41). First, a simplified expression of $s(t, \underline{w})$ can be obtained by observing that

$$
\begin{aligned}
\underline{\underline{C}}_{0}^{-1}: \underline{\underline{e}}_{p}(\underline{w})-2 \underline{\underline{C}}_{0}^{-1} \cdot \underline{\underline{e}}_{0}\left(\underline{y}_{p}\right) \cdot \underline{\underline{C}}_{0}^{-1}: \underline{\underline{e}}_{0}(\underline{w}) & =\left(\underline{\underline{\nabla}} \underline{y}_{p} \underline{\underline{C}}_{0}^{-1}-2 \underline{\underline{F}}_{0}^{-T} \cdot \underline{\underline{e}}_{0}\left(\underline{y}_{p}\right) \cdot \underline{\underline{C}}_{0}^{-1}\right): \underline{\underline{\nabla}} \underline{\underline{w}} \\
& =\left(\underline{\underline{\nabla}} \underline{y}_{p} \underline{\underline{F}}_{0}^{-1}-2 \underline{\underline{F}}_{0}^{-T} \cdot \underline{\underline{e}}_{0}\left(\underline{y}_{p}\right) \cdot \underline{\underline{F}}_{0}^{-1}\right) \cdot \underline{\underline{F}}_{0}^{-T}: \underline{\underline{\nabla}} \underline{\underline{w}} \\
& =-\underline{\underline{F}}_{0}^{-T} \cdot\left(\underline{\underline{\nabla}} \underline{\underline{y}}_{p}\right)^{T} \cdot \underline{\underline{F}}_{0}^{-T}: \underline{\underline{\nabla}} \underline{w} .
\end{aligned}
$$

Therefore, inserting the expression above into Eq. (5.42) and using Green's formula (as in Eq. (5.32)) and Eq. (5.33), one can see that

$$
s(t, \underline{w})=-\frac{\hat{\omega}}{2 \pi} \int_{0}^{\frac{2 \pi}{\hat{\omega}}} \int_{\Omega_{0}} \underline{\operatorname{div}}\left(\left(1+\hat{\zeta} \partial_{\tau}\right) \operatorname{tr}\left(\underline{\underline{C}}_{0}^{-1} \cdot \underline{\underline{e}}_{0}\left(\underline{y}_{p}\right)\right) \underline{\underline{F}}_{0}^{-T} \cdot\left(\underline{\underline{\nabla}} \underline{y}_{p}\right)^{T} \cdot \underline{\underline{F}}_{0}^{-T}\right) \cdot \underline{w} \mathrm{~d} \Omega_{0} \mathrm{~d} \tau+b(t, w),
$$


with

$$
b(t, w)=\frac{\hat{\omega}}{2 \pi} \int_{0}^{\frac{2 \pi}{\hat{\omega}}} \int_{\Gamma_{0}} g\left(\underline{\mathrm{t}}_{2}+\underline{\underline{F}}_{0}^{-T} \cdot\left(\underline{\underline{\nabla}} \underline{y}_{p}\right)^{T} \cdot \underline{\underline{F}}_{0}^{-T} \cdot \underline{n}_{0}\right) \cdot \underline{w} \mathrm{~d} \Gamma_{0} \mathrm{~d} \tau .
$$

Moreover, one can show that

$$
\underline{\mathrm{t}}_{2}=J_{2} \underline{\underline{F}}_{0}^{-T} \cdot \underline{n}_{0}-\underline{\underline{F}}_{0}^{-T} \cdot\left(\underline{\underline{\nabla}} \underline{y}_{2}\right)^{T} \cdot \underline{\underline{F}}_{0}^{-T} \cdot \underline{n}_{0},
$$

hence, using again the property that $\underline{y}_{2}=\underline{y}_{p}+\underline{y}_{s}$ and $\underline{y}_{s}$ is independent of $\tau$, the boundary term $b(t, w)$ reads

$$
b(t, w)=\frac{\hat{\omega}}{2 \pi} \int_{0}^{\frac{2 \pi}{\hat{\omega}}} \int_{\Gamma_{0}} g J_{2} \underline{w} \cdot \underline{\underline{F}}_{0}^{-T} \cdot \underline{n}_{0} \mathrm{~d} \Gamma_{0} \mathrm{~d} \tau=\int_{\Gamma_{0}}\left(\frac{\hat{\omega}}{2 \pi} \int_{0}^{\frac{2 \pi}{\hat{\omega}}} g p \mathrm{~d} \tau\right) \underline{w} \cdot \underline{\underline{F}}_{0}^{-T} \cdot \underline{n}_{0} \mathrm{~d} \Gamma_{0},
$$

with $p=\operatorname{tr}\left(\underline{\underline{C}}_{0}^{-1} \cdot \underline{\underline{e}}_{0}\left(\underline{y}_{p}\right)\right)$. Using Eq. (5.33), one can check that $\hat{\zeta} \partial_{\tau} p+p=g$. Consequently,

$$
\int_{0}^{\frac{2 \pi}{\hat{\omega}}} g p \mathrm{~d} \tau=\int_{0}^{\frac{2 \pi}{\hat{\omega}}} p\left(1+\hat{\zeta} \partial_{\tau}\right) p \mathrm{~d} \tau=\int_{0}^{\frac{2 \pi}{\hat{\omega}}} p^{2} \mathrm{~d} \tau .
$$

One can rewrite Eq. (5.43) in the deformed configuration and show that (5.40) holds, thus concluding the proof (note that we have used the property $\left(\underline{\underline{\nabla}}_{\underline{x}} \underline{y}_{p}\right)^{T}=\underline{\underline{\nabla}}_{\underline{x}} \underline{y}_{p}$ that results from Eq. (5.31)).

Remark We can see that the term (5.40) involves a nonlinear (quadratic) contribution only depending on $\underline{y}_{p}$. Thus, it is a source term (accounting for the ARF contribution) and, although $\underline{y}_{p}$ has zero mean in $\tau$, it gives a non-zero contribution in the equation, due to nonlinearity.

Remark Note that $\mathrm{p}$ represents a Lagrange multiplier for the solution $\underline{y}_{s}$. We also emphasise that, if the solution of the nonlinear problem $\underline{y}_{0}$ is zero, then the Lagrangian multiplier $\mathrm{p}_{0}$ is also zero, which implies that in this case the term $\underline{y}_{s}$ is divergence-free.

\subsection{Proof of Corollary 4.2}

If we assume that the boundary source term $g$ is a combination of sine and cosine function at a single frequency, e.g.,

$$
g(\underline{\xi}, t, \tau)=\hat{p}_{c}(\underline{\xi}, t) \cos (\hat{\omega} \tau)+\hat{p}_{s}(\underline{\xi}, t) \sin (\hat{\omega} \tau),
$$

then one can further simplify the expression of the ARF. To do so, we reduce System (5.31) to a frequency problem on the pressure variable $p$. The scalar field $p=\operatorname{div}_{\underline{x}} \underline{y}_{p}$ satisfies

$$
p=\operatorname{Re}\left(\hat{p} e^{-i \hat{\omega} \tau}\right)
$$


where $\hat{p}$ is solution of

$$
\begin{cases}\Delta_{\underline{x}} \hat{p}+\alpha \hat{p}=0 & \text { in } \quad \Omega(t) \\ (1-i \hat{\omega} \hat{\zeta}) \hat{p}(\underline{x}, t)=\hat{p}_{c}+i \hat{p}_{s} & \text { on } \quad \Gamma(t)\end{cases}
$$

with

$$
\alpha:=\frac{\hat{\rho}_{0} \hat{\omega}^{2}}{1-i \hat{\omega} \hat{\zeta}} \in \mathbb{C} .
$$

Eq. (5.47) represents for each time $t$ a Helmholtz equation governing the propagation of the pushing pressure wave corresponding to the ARF. Note that $t$ plays the role of a parameter in this equation, due to Eq. (5.46). Problem (5.47) is well-posed, for all time $t$, because $\operatorname{Im}(\alpha) \neq 0$. To continue in the simplification process we introduce the function $\underline{\hat{y}}_{p}$ such that $\underline{\hat{y}}_{p}=-\alpha^{-1} \underline{\nabla}_{\underline{x}} \hat{p}$. By definition of $\hat{p}$, we retrieve that $\operatorname{div}_{\underline{x}} \underline{\hat{y}}_{p}=\hat{p}$. In addition, the function $\underline{y}_{p}$ satisfies

$$
\underline{y}_{p}=\operatorname{Re}\left(\underline{\hat{y}}_{p} e^{-i \hat{\omega} \tau}\right) \quad \text { and } \quad\left(1+\hat{\zeta} \partial_{\tau}\right) \underline{y}_{p}=\operatorname{Re}\left((1-i \hat{\omega} \hat{\zeta}) \underline{y}_{p} e^{-i \hat{\omega} \tau}\right)=\hat{\rho}_{0} \hat{\omega}^{2} \operatorname{Re}\left(\alpha^{-1} \underline{y}_{p} e^{-i \hat{\omega} \tau}\right) .
$$

As an intermediate step towards the proof of Corollary 4.2, we state the following proposition that enables to deduce an alternative expression of the source term $s(t, \underline{w})$ of Proposition 5.6.

Proposition 5.7. The following equalities hold:

$$
\frac{\hat{\omega}}{2 \pi} \int_{0}^{\frac{2 \pi}{\hat{\omega}}} \operatorname{div}_{\underline{x}}\left(\left(1+\hat{\zeta} \partial_{\tau}\right) \underline{y}_{p}\right) \underline{\underline{\nabla}}_{\underline{x}} \underline{y} \mathrm{~d} \tau=-\frac{\beta}{2} \operatorname{Re}\left(\hat{\hat{p}} \underline{\underline{H}}_{\underline{x}}(\overline{\hat{p}})\right)
$$

and

$$
\underline{\operatorname{div}}_{\underline{x}} \beta \operatorname{Re}\left(\hat{p} \underline{\underline{H}}_{\underline{x}}(\overline{\hat{p}})\right)=\frac{\beta}{2} \underline{\nabla}_{\underline{x}}\left|\underline{\nabla}_{\underline{x}} \hat{p}\right|^{2}-\frac{1}{2} \underline{\nabla}_{\underline{x}}|\hat{p}|^{2}-\hat{\zeta} \hat{\omega} \operatorname{Im}\left(\hat{p} \underline{\nabla}_{\underline{x}} \overline{\hat{p}}\right),
$$

with

$$
\beta:=\operatorname{Re}(\bar{\alpha})^{-1}=\frac{\hat{\rho}_{0} \hat{\omega}^{2}}{|\alpha|^{2}}=\frac{|1-i \hat{\omega} \hat{\zeta}|^{2}}{\hat{\rho}_{0} \hat{\omega}^{2}} .
$$

Proof. Observe that, using (5.49), we can write

$$
S:=\frac{\hat{\omega}}{2 \pi} \int_{0}^{\frac{2 \pi}{\hat{\omega}}} \operatorname{div}_{\underline{x}}\left(\left(1+\hat{\zeta} \partial_{\tau}\right) \underline{y}_{p}\right) \underline{\underline{\nabla}}_{\underline{x}} \underline{y}_{p} \mathrm{~d} \tau=\rho_{0} \hat{\omega}^{2} \frac{\hat{\omega}}{2 \pi} \int_{0}^{\frac{2 \pi}{\hat{\omega}}} \operatorname{Re}\left(\alpha^{-1} \operatorname{div}_{\underline{x}} \underline{\hat{y}}_{p} e^{-i \hat{\omega} \tau}\right) \operatorname{Re}\left(\underline{\underline{\nabla}}_{\underline{x}} \underline{\hat{y}}_{p} e^{-i \hat{\omega} \tau}\right) \mathrm{d} \tau .
$$

However, since for any $(a, b) \in \mathbb{C}^{2}$ we have

$$
\frac{\hat{\omega}}{2 \pi} \int_{0}^{\frac{2 \pi}{\hat{\omega}}} \operatorname{Re}\left(a e^{-i \hat{\omega} \tau}\right) \operatorname{Re}\left(b e^{-i \hat{\omega} \tau}\right) \mathrm{d} \tau=\frac{1}{2} \operatorname{Re}(a \bar{b}),
$$

by extension of this property and using the definition of $\hat{p}$, one can show that

$$
S=\frac{\rho_{0} \hat{\omega}^{2}}{2} \operatorname{Re}\left(\alpha^{-1} \operatorname{div}_{\underline{x}} \underline{\hat{y}}_{p} \underline{\underline{\nabla}}_{\underline{x}} \underline{\hat{\hat{y}}}_{p}\right)=-\frac{\beta}{2} \operatorname{Re}\left(\hat{p} \underline{\underline{H}}_{\underline{x}}(\overline{\hat{p}})\right),
$$


that corresponds to the first equality to be proved. In order to prove the second equality, observe that

$$
\underline{\operatorname{div}}_{\underline{x}}\left(\hat{p} \underline{\underline{H}}_{\underline{x}}(\overline{\hat{p}})\right)=\underline{\underline{H}}_{\underline{x}}(\overline{\hat{p}}) \cdot \underline{\nabla}_{\underline{x}} \hat{p}+\hat{p} \underline{\nabla}_{\underline{x}} \Delta_{\underline{x}} \overline{\hat{p}}=V+W
$$

with

$$
V:=\underline{\underline{H}}_{\underline{x}}(\overline{\hat{p}}) \cdot \underline{\nabla}_{\underline{x}} \hat{p}, \quad W:=\hat{p} \underline{\nabla}_{\underline{x}} \Delta_{\underline{x}} \overline{\hat{p}}=-\bar{\alpha} \hat{p} \underline{\nabla}_{\underline{x}} \overline{\hat{p}} .
$$

Only the real part of $V$ and $W$ needs to be specified. We have

$$
\operatorname{Re}(\underline{V})=\underline{\underline{H}}_{\underline{x}}(\operatorname{Re}(\hat{p})) \cdot \underline{\nabla}_{\underline{x}} \operatorname{Re}(\hat{p})+\underline{\underline{H}}_{\underline{x}}(\operatorname{Im}(\hat{p})) \cdot \underline{\nabla}_{\underline{x}} \operatorname{Im}(\hat{p}) .
$$

Now, since

$$
\underline{\underline{H}}_{\underline{x}}(\operatorname{Re}(\hat{p})) \cdot \underline{\nabla}_{\underline{x}} \operatorname{Re}(\hat{p})=\sum_{j} \frac{\partial^{2} \operatorname{Re}(\hat{p})}{\partial x_{i} \partial x_{j}} \frac{\partial \operatorname{Re}(\hat{p})}{\partial x_{j}}=\frac{1}{2} \sum_{j} \underline{\nabla}_{\underline{x}}\left(\frac{\partial \operatorname{Re}(\hat{p})}{\partial x_{j}}\right)^{2}
$$

and analogously for $\underline{\underline{H}}_{\underline{x}}(\operatorname{Im}(\hat{p})) \cdot \underline{\nabla}_{\underline{x}} \operatorname{Im}(\hat{p})$, we can simplify $V$ as

$$
\operatorname{Re}(V)=\frac{1}{2} \sum_{j} \underline{\nabla}_{\underline{x}}\left|\partial_{x_{j}} \hat{p}\right|^{2}=\frac{1}{2} \underline{\nabla}_{\underline{x}}\left|\underline{\nabla}_{\underline{x}} \hat{p}\right|^{2} .
$$

On the other hand, since $\operatorname{Re}(\bar{\alpha})=\beta^{-1}$ and $\operatorname{Im}(\bar{\alpha})=-\beta^{-1} \hat{\zeta} \hat{\omega}$ we can express

$$
\beta \operatorname{Re}(W)=-\operatorname{Re}\left(\hat{p} \underline{\nabla}_{\underline{x}}(\overline{\hat{p}})\right)-\hat{\zeta} \hat{\omega} \operatorname{Im}\left(\hat{p} \underline{\nabla}_{\underline{x}}(\overline{\hat{p}})\right) .
$$

Since $2 \operatorname{Re}\left(\hat{p} \underline{\nabla}_{\underline{x}} \overline{\hat{p}}\right)=\underline{\nabla}_{\underline{x}}|\hat{p}|^{2}$ we finally have

$$
\beta \operatorname{Re}(W)=-\frac{1}{2} \underline{\nabla}_{\underline{x}}|\hat{p}|^{2}-\hat{\zeta} \hat{\omega} \operatorname{Im}\left(\hat{p} \underline{\nabla}_{\underline{x}}(\overline{\hat{p}})\right) .
$$

Collecting the two results above, we obtain the second equality of the statement.

Proposition 5.8. There exists another scalar field $\tilde{\mathrm{p}}$ such that the couple $\left(\underline{y}_{s}, \tilde{\mathrm{p}}\right)$ satisfies Eq. (3.3) with

$$
s(t, \underline{w})=\int_{\Omega(t)} \underline{F}_{A R F} \cdot \underline{w} \mathrm{~d} \Omega+\int_{\Gamma_{N}(t)} \underline{w} \cdot \underline{\tilde{\sigma}}_{b n d y} \cdot \underline{n} \mathrm{~d} \Gamma, \quad r(t, q)=\int_{\Omega_{0}} \mathrm{p}_{0} q \mathrm{~d} \Omega_{0},
$$

where $\underline{F}_{A R F}$ is defined as

$$
\underline{F}_{A R F}=-\frac{\hat{\zeta} \hat{\omega}}{2} \operatorname{Im}\left(\hat{p} \underline{\nabla}_{\underline{x}} \overline{\hat{p}}\right)
$$

and $\underline{\underline{\sigma}}_{b n d y}$ is given by

$$
\underline{\underline{\tilde{\sigma}}}_{b n d y}=\frac{1}{4}\left(\beta\left|\underline{\nabla}_{\underline{x}} \hat{p}\right|^{2}+|\hat{p}|^{2}\right) \underline{\mathbb{1}} .
$$


Proof. From the first and second equality of Corollary 5.7 we have that the source term $s(t, \underline{w})$ given in Proposition 5.6 satisfies, for all real test function $\underline{w}$

$$
s(t, \underline{w})=\int_{\Omega(t)}\left(\frac{\beta}{4} \underline{\nabla}_{\underline{x}}\left|\underline{\nabla}_{\underline{x}} \hat{p}\right|^{2}-\frac{1}{4} \underline{\nabla}_{\underline{x}}|\hat{p}|^{2}-\frac{\hat{\zeta} \hat{\omega}}{2} \operatorname{Im}\left(\hat{p} \underline{\nabla}_{\underline{x}} \hat{\hat{p}}\right)\right) \cdot \underline{w} \mathrm{~d} \Omega+\frac{1}{2} \int_{\Gamma(t)}|\hat{p}|^{2} \underline{w} \cdot \underline{n} \mathrm{~d} \Gamma \mathrm{d} \tau,
$$

where we have used the property that

$$
\frac{\hat{\omega}}{2 \pi} \int_{0}^{\frac{2 \pi}{\hat{\omega}}} p^{2} \mathrm{~d} \tau=\frac{|\hat{p}|^{2}}{2} .
$$

Using Green's formula in (5.55), one can write

$$
s(t, \underline{w})=\int_{\Omega(t)} \underline{F}_{A R F} \cdot \underline{w} \mathrm{~d} \Omega+\int_{\Gamma_{N}(t)} \underline{w} \cdot \underline{\underline{\sigma}}_{b n d y} \cdot \underline{n} \mathrm{~d} \Gamma-\int_{\Omega(t)} \breve{\mathrm{p}} \operatorname{div}_{\underline{x}} \underline{w} \mathrm{~d} \Omega,
$$

where $\underline{F}_{A R F}$ and $\underline{\tilde{\sigma}}_{b n d y}$ are given by (5.53) and (5.54) respectively, while

$$
\breve{\mathrm{p}}=\frac{\beta}{4}\left|\underline{\nabla}_{\underline{x}} \hat{p}\right|^{2}-\frac{1}{4}|\hat{p}|^{2}
$$

Finally, since

$$
\int_{\Omega(t)} \breve{\mathrm{p}} \operatorname{div}_{\underline{x}} \underline{w} \mathrm{~d} \Omega=\int_{\Omega_{0}} \check{\mathrm{p}} \underline{\underline{C}}_{0}^{-1}: \underline{\underline{e}}_{0}(\underline{w}) \mathrm{d} \Omega_{0},
$$

one can check that the couple $\left(\underline{y}_{s}, \tilde{\mathrm{p}}\right)$ with $\tilde{\mathrm{p}}=\mathrm{p}+\check{\mathrm{p}}$ satisfies Eq. (3.3) with $s(t, \underline{w})$ and $r(t, q)$ as in the statement of the Proposition.

\section{Conclusion}

We have derived an original mathematical model of the acoustic radiation force phenomenon in a nonlinear, nearly-incompressible solid. We emphasise that the proposed model allows to dispense with the simplifying hypotheses that are usually made on the properties of the solution (e.g. plane wave assumption).

In greater detail, based on energy considerations and asymptotic analysis, we have derived the governing equation of the pressure field and the shear wave field remotely induced by the ARF and we have computed an analytical expression of the source term responsible for the generation of shear waves from an acoustic pressure pulse. As a by-product of our analysis, we have shown that it is associated with the viscosity and the tissue nonlinearities. Moreover, note that the analytical expression of the source term responsible for shear wave propagation that we have provided is, to the best of our knowledge, the first mathematical formulation of the ARF field in weak formulation, and it is very suitable for a Finite Element framework. This work opens numerous perspectives: concerning the analysis, one could try to make the derivation of the ARF less formal by proving the results presented (e.g. by adding global viscosity to the problems to 
ease the mathematical analysis); from the modelling point of view, first, one could study more involved expressions of the boundary source term in order to take into account, for instance, modulation effects or nonlinear deformation effects due to the force exerted by the probes on the tissue, second, one could consider more advanced viscosity models and analyse the influence of such choices; from a numerical perspective, a numerical scheme for the whole process could be constructed, either to validate the formal asymptotic analysis and/or to investigate the ARF (in particular the effect of the boundary excitation); finally, comparisons with other derivations of the ARF expressions could be carried out in order to understand the range of validity of such expressions.

\section{Appendix A Proof of Lemma 2.1}

Positivity properties of the elastic potentials. As preliminary results we study some properties of the non-dimensionalised elastic potentials $W^{\mathrm{NI}}$ and $W^{\mathrm{TI}}$ used to define the total energy of the system. In this paragraph $\underline{\underline{e}}=\left(\underline{\underline{F}}^{T} \underline{\underline{F}}-\underline{\underline{\mathbb{1}}}\right) / 2$ represents any Green-Lagrange tensor associated to a deformation gradient $\underline{\underline{F}}$. We have $J=\operatorname{det} \underline{\underline{F}}$ and the invariants $I_{1}, I_{2}$ and $I_{4}$ are given by the formulae in Eq. (2.8).

\section{Proposition A.1.}

$$
W^{N I}(\underline{\underline{e}}) \geq 0 \quad \text { and } \quad W^{N I}(\underline{\underline{e}}) \geq \frac{1}{8} J^{2}-\frac{1}{4} .
$$

Proof. By definition we have $4 W^{\mathrm{NI}}(\underline{\underline{e}}):=\left(J^{2}-1\right)-\log \left(J^{2}\right)$, hence, one can easily show that this function of $J^{2}$ is positive. Moreover, since $\log x \leq x / e$ for $x \geq 0$, we have

$$
4 W^{\mathrm{NI}}(\underline{\underline{e}})=\left(J^{2}-1\right)-\log \left(J^{2}\right) \geq\left(J^{2}-1\right)-J^{2} / e,
$$

and therefore we deduce

$$
W^{\mathrm{NI}}(\underline{\underline{e}}) \geq \frac{1}{4}\left(J^{2}-1\right)-\frac{J^{2}}{4 e}=\frac{e-1}{4 e} J^{2}-\frac{1}{4} .
$$

The result of the Proposition follows from the property $(e-1) / e>1 / 2$.

\section{Proposition A.2.}

$$
W^{T I}(\underline{\underline{e}})+8 \hat{\kappa}_{3} W^{N I}(\underline{\underline{e}}) \geq \hat{\kappa}_{1} e^{\hat{\eta}_{1}\left(I_{1}-3\right)^{2}}+\hat{\kappa}_{2} e^{\hat{\eta}_{2}\left(I_{4}-1\right)^{2}}+\hat{\kappa}_{3} I_{2}-5 \hat{\kappa}_{3},
$$

Proof. We first recall the definition of the potential $W^{\mathrm{TI}}$. It reads

$$
W^{\mathrm{TI}}(\underline{\underline{e}})=\hat{\kappa}_{1} e^{\hat{\eta}_{1}\left(I_{1}-3\right)^{2}}+\hat{\kappa}_{2} e^{\hat{\eta}_{2}\left(I_{4}-1\right)^{2}}+\hat{\kappa}_{3}\left(I_{2}-2 \log \left(J^{2}\right)-3\right) .
$$

We have, since $\log x \leq x / e<x / 2$ for $x \geq 0$ and thanks to Proposition A.1,

$$
W^{\mathrm{TI}}(\underline{\underline{e}})+8 \hat{\kappa}_{3} W^{\mathrm{NI}}(\underline{\underline{e}}) \geq \hat{\kappa}_{1} e^{\hat{\eta}_{1}\left(I_{1}-3\right)^{2}}+\hat{\kappa}_{2} e^{\hat{\eta}_{2}\left(I_{4}-1\right)^{2}}+\hat{\kappa}_{3}\left(I_{2}-J^{2}-3\right)+\hat{\kappa}_{3} J^{2}-2 \hat{\kappa}_{3},
$$

from which estimation (A.1) follows. 
Energy identity for the parametrised family of problems. Let Assumption 2.1 hold, then, choosing the velocity $\partial_{t} \underline{y}_{\varepsilon}$ as a test function in Eq. (2.17), we obtain the following energy balance (see [21])

$$
\frac{\mathrm{d} \varepsilon_{\varepsilon}^{t o t}}{\mathrm{~d} t}+\mathcal{P}_{\varepsilon}^{\mathrm{V}}=\mathcal{P}_{\varepsilon}^{e x t}+\mathcal{P}_{\varepsilon}^{A}
$$

where the total energy $\mathcal{E}_{\varepsilon}^{\text {tot }}$ reads

$$
\varepsilon_{\varepsilon}^{t o t}=\mathcal{K}_{\varepsilon}+\varepsilon_{\varepsilon}^{\mathrm{TI}}+\varepsilon_{\varepsilon}^{\mathrm{NI}}
$$

and the kinetic and potential energy associated with the hyperelastic term and near-incompressibility are given by

$$
\mathcal{K}_{\varepsilon}=\frac{\hat{\rho}_{0}}{2} \int_{\Omega_{0}}\left|\partial_{t} \underline{y}\right|^{2} \mathrm{~d} \Omega_{0}, \quad \varepsilon_{\varepsilon}^{\mathrm{TI}}=\int_{\Omega_{0}} W^{\mathrm{TI}}(\underline{\underline{e}}) \mathrm{d} \Omega_{0}, \quad \varepsilon_{\varepsilon}^{\mathrm{NI}}=\varepsilon^{-2} \int_{\Omega_{0}} W^{\mathrm{NI}}\left(\underline{\underline{e}}_{\varepsilon}\right) \mathrm{d} \Omega_{0} .
$$

The contribution related to viscous losses reads

$$
\mathcal{P}_{\varepsilon}^{\mathrm{V}}=2 \varepsilon^{-1} \hat{\zeta} \int_{\Omega_{0}} V\left(\underline{\underline{e}}_{\varepsilon}, \underline{\underline{\dot{e}}}{ }_{\varepsilon}\right) \mathrm{d} \Omega_{0}=2 \varepsilon^{-1} \hat{\zeta} \int_{\Omega_{0}} \operatorname{tr}\left(\underline{\underline{C}}_{\varepsilon}^{-1} \cdot \underline{\underline{\dot{e}}}_{\varepsilon}\right)^{2} \mathrm{~d} \Omega_{0}=2 \varepsilon^{-1} \hat{\zeta} \int_{\Omega_{0}} \operatorname{tr}\left(\underline{\underline{F}}_{\varepsilon}^{-1} \cdot \underline{\underline{\nabla}} \partial_{t} \underline{y}_{\varepsilon}\right)^{2} \mathrm{~d} \Omega_{0},
$$

whereas the source term contributions are given by

$$
\mathcal{P}_{\varepsilon}^{e x t}=\int_{\Gamma_{0}} \underline{\mathrm{t}}_{\varepsilon} \cdot \partial_{t} \underline{y}_{\varepsilon} \mathrm{d} \Gamma_{0}=\int_{\Gamma_{0}} g_{\varepsilon} J_{\varepsilon} \partial_{t} \underline{y}_{\varepsilon} \cdot \underline{\underline{F}}_{\varepsilon}^{-T} \cdot \underline{n}_{0} \mathrm{~d} \Gamma_{0}
$$

with $g \equiv g(\underline{\xi}, t, t / \varepsilon)$ and

$$
\mathcal{P}_{\varepsilon}^{A}=-\int_{\Omega_{0}} \underline{\Sigma}^{A}: \underline{\underline{e}}_{\varepsilon} \mathrm{d} \Omega_{0} .
$$

Coercivity of the energy functional. From the positivity results of the functional $W^{\mathrm{NI}}$ and $W^{\mathrm{TI}}$ we are able to deduce a type of coercivity of the potential energy associated to smooth solutions of $(2.17)$.

Proposition A.3. There exists a dimensionless scalar $C>0$ such that, for all $\varepsilon \leq 8 \hat{\kappa}_{3}+1$, it holds

$$
\int_{\Omega_{0}}\left|\partial_{t} \underline{y}_{\varepsilon}\right|^{2}+\int_{\Omega_{0}}\left(I_{1, \varepsilon}+I_{1, \varepsilon}^{2}\right) \mathrm{d} \Omega_{0}+\int_{\Omega_{0}}\left(I_{4, \varepsilon}+I_{4, \varepsilon}^{2}\right) \mathrm{d} \Omega_{0}+\int_{\Omega_{0}} I_{2, \varepsilon} \mathrm{d} \Omega_{0}+\int_{\Omega_{0}} J_{\varepsilon}^{2} \mathrm{~d} \Omega_{0} \leq \mathcal{E}_{\varepsilon}^{t o t}+C
$$

where

$$
I_{1, \varepsilon}=\operatorname{tr}(\underline{\underline{C}}), \quad I_{2, \varepsilon}=|\operatorname{adj} \underline{\underline{F}}|^{2}, \quad I_{4, \varepsilon}=\underline{\tau} \cdot \underline{\underline{C}} \cdot \underline{\tau} .
$$

Proof. We write the decomposition

$$
\mathcal{E}_{\varepsilon}^{t o t}=\mathcal{K}_{\varepsilon}+\int_{\Omega_{0}}\left(W^{\mathrm{TI}}\left(\underline{\underline{e}}_{\varepsilon}\right)+8 \hat{\kappa}_{3} W^{\mathrm{NI}}\left(\underline{\underline{e}}_{\varepsilon}\right)\right) \mathrm{d} \Omega_{0}+\int_{\Omega_{0}} W^{\mathrm{NI}}\left(\underline{\underline{e}}_{\varepsilon}\right) \mathrm{d} \Omega_{0}+\varepsilon^{2}\left(\varepsilon^{-2}-8 \hat{\kappa}_{3}-1\right) \mathcal{E}_{\varepsilon}^{\mathrm{NI}} .
$$


Therefore, since $\varepsilon \leq 8 \hat{\kappa}_{3}+1$ and $\mathcal{E}_{\varepsilon}^{\mathrm{NI}} \geq 0$ we deduce that

$$
\mathcal{K}_{\varepsilon}+\left(\varepsilon_{\varepsilon}^{\mathrm{TI}}+8 \hat{\kappa}_{3} \varepsilon_{\varepsilon}^{\mathrm{TI}}\right)+\varepsilon_{\varepsilon}^{\mathrm{NI}} \leq \varepsilon_{\varepsilon}^{t o t} .
$$

Using Propositions A.1 and A.2 we get

$$
\mathcal{K}_{\varepsilon}+\int_{\Omega_{0}}\left(\hat{\kappa}_{1} e^{\hat{\eta}_{1}\left(I_{1, \varepsilon}-3\right)^{2}}+\hat{\kappa}_{2} e^{\hat{\eta}_{2}\left(I_{4, \varepsilon}-1\right)^{2}}+\hat{\kappa}_{3} I_{2, \varepsilon}+\frac{1}{4} J_{\varepsilon}^{2}\right) \mathrm{d} \Omega_{0} \leq \mathcal{E}_{\varepsilon}^{t o t}+\left(5 \hat{\kappa}_{3}+\frac{1}{2}\right)\left|\Omega_{0}\right| .
$$

Observe that $e^{x} \geq x$ and $6 I_{1, \varepsilon} \leq I_{1, \varepsilon}^{2} / 2+18$, we get

$$
e^{\hat{\eta}_{1}\left(I_{1, \varepsilon}-1\right)^{2}} \geq \hat{\eta}_{1}\left(I_{1, \varepsilon}-3\right)^{2} \geq \frac{\hat{\eta}_{1}}{2} I_{1, \varepsilon}^{2}-9 \hat{\eta}_{1}
$$

Similarly, noting that $e^{\eta_{*} x^{2}} \geq|x|$ with $\eta_{*}=1 /(2 e)$, one can deduce that

$$
e^{\hat{\eta}_{1}\left(I_{1, \varepsilon}-3\right)^{2}} \geq \frac{\sqrt{\hat{\eta}_{1}}}{\sqrt{\eta_{*}}}\left|I_{1, \varepsilon}-3\right| \geq \frac{\sqrt{\hat{\eta}_{1}}}{\sqrt{\eta_{*}}} I_{1, \varepsilon}-3 \frac{\sqrt{\hat{\eta}_{1}}}{\sqrt{\eta_{*}}} .
$$

In the same way, one can show that

$$
e^{\hat{\eta}_{2}\left(I_{4, \varepsilon}-1\right)^{2}} \geq \frac{\hat{\eta}_{2}}{2} I_{4, \varepsilon}^{2}-\hat{\eta}_{2} \quad \text { and } \quad e^{\hat{\eta}_{2}\left(I_{4, \varepsilon}-1\right)^{2}} \geq \frac{\sqrt{\hat{\eta}_{2}}}{\sqrt{\eta_{*}}} I_{4, \varepsilon}-\frac{\sqrt{\hat{\eta}_{2}}}{\sqrt{\eta_{*}}}
$$

The result of the Proposition is deduced by using the estimations (A.4, A.5, A.6) into (A.3).

Estimation of the surface source term. As a first preliminary estimation, we derive an estimate on $\mathcal{P}_{\varepsilon}^{\text {ext }}$, that we rewrite as a volume integral. Because of the regularity assumption on the domain $\Omega_{0}$ and $g$, one can assume that there exists a smooth function $\ell(\underline{\xi}, t, t / \varepsilon)$ defined in $\Omega_{0} \times[0, T] \times[0,2 \pi / \hat{\omega}]$ such that

$$
\forall(t, \tau) \in[0, T] \times\left.[0,2 \pi / \hat{\omega}] \quad \ell(\cdot, t, \tau)\right|_{\Gamma_{0}}=g(\cdot, t, \tau) .
$$

Then we take into account Eq. (A.7), and rewrite $\mathcal{P}_{\varepsilon}^{e x t}$ as

$$
\mathcal{P}_{\varepsilon}^{e x t}(t)=\int_{\Gamma_{0}} \ell J_{\varepsilon} \partial_{t} \underline{y}_{\varepsilon} \cdot \underline{F}_{\varepsilon}^{-T} \cdot \underline{n}_{0} \mathrm{~d} \Gamma_{0} .
$$

Contrarily to what was stated at the beginning of Section 2 we now consider Eq. (A.8) in the deformed configuration obtained with the displacement $\underline{y}_{\varepsilon}$ (and not $\underline{y}_{0}$ ) in order to use Eq. (2.1). Therefore we introduce the deformation map $\underline{\phi}_{\varepsilon}$ and the deformed domain $\Omega_{\varepsilon}(t)$ such that $\underline{\phi}_{\varepsilon}: \bar{\Omega}_{0} \rightarrow \Omega_{\varepsilon}(t)$ with $\underline{\phi}_{\varepsilon}(\underline{\xi}, t)=\underline{y}_{\varepsilon}(\underline{\xi}, t)+\underline{\xi}$. Then, using Eq. (A.8) and applying the divergence theorem, we have

$$
\mathcal{P}_{\varepsilon}^{e x t}(t)=\int_{\partial \Omega_{\varepsilon}} \ell \partial_{t} \underline{y}_{\varepsilon} \cdot \underline{n} \mathrm{~d} \Gamma=\int_{\Omega_{\varepsilon}(t)} \underline{\nabla}_{\underline{x}} \cdot \partial_{t} \underline{y}_{\varepsilon} \ell \mathrm{d} \Omega+\int_{\Omega_{\varepsilon}(t)} \partial_{t} \underline{y}_{\varepsilon} \cdot \underline{\nabla}_{\underline{x}} \ell \mathrm{d} \Omega .
$$


Eq. (A.9) can be rewritten in Lagrangian formulation using Eq. (2.1), leading to

$$
\mathcal{P}_{\varepsilon}^{e x t}(t)=\int_{\Omega_{0}} J_{\varepsilon} \operatorname{tr}\left(\underline{\underline{F}}_{\varepsilon}^{-1} \cdot \underline{\underline{\nabla}} \partial_{t} \underline{\underline{y}}\right) \ell \mathrm{d} \Omega_{0}+\int_{\Omega_{0}} J_{\varepsilon} \underline{\underline{F}}_{\varepsilon}^{-T} \cdot \underline{\nabla} \ell \cdot \partial_{t} \underline{\underline{y}} \mathrm{~d} \Omega_{0} .
$$

Using now Young's inequality on Eq. (A.10) one can show that

$$
\begin{aligned}
\mathcal{P}_{\varepsilon}^{e x t}(t) \leq \frac{\sup _{\underline{\xi} \in \Omega_{0}}|\ell(\xi, t, t / \varepsilon)|}{2} \int_{\Omega_{0}}\left(J_{\varepsilon}^{2}+\operatorname{tr}\left(\underline{\underline{F}}_{\varepsilon}^{-1} \cdot \underline{\underline{\nabla}} \partial_{t} \underline{y}_{\varepsilon}\right)^{2}\right) \mathrm{d} \Omega_{0} & \\
& +\frac{\sup _{\underline{\xi} \in \Omega_{0}}|\underline{\nabla} \ell(\xi, t, t / \varepsilon)|}{2} \int_{\Omega_{0}}\left(I_{2, \varepsilon}+\left|\partial_{t} \underline{y}_{\varepsilon}\right|^{2}\right) \mathrm{d} \Omega_{0},
\end{aligned}
$$

where we have used the definition that $\operatorname{adj} \underline{\underline{F}}_{\varepsilon}=J_{\varepsilon} \underline{\underline{F}}_{\varepsilon}^{-1}$ and $I_{2, \varepsilon}=\left|\operatorname{adj} \underline{\underline{F}}_{\varepsilon}\right|^{2}$. Since $\ell$ and $\underline{\nabla} \ell$ have an amplitude independent of $\varepsilon$, one can show, using Proposition A.3, that there exists a dimensionless scalar $C>0$ such that

$$
\int_{0}^{t} \mathcal{P}_{\varepsilon}^{e x t}(s) \mathrm{d} s \leq C\left(1+\varepsilon \int_{0}^{t} \mathcal{P}_{\varepsilon}^{\mathrm{V}}(s) \mathrm{d} s+\int_{0}^{t} \mathcal{E}_{\varepsilon}^{t o t}(s) \mathrm{d} s\right)
$$

Estimation of the volumic source terms. We now give an estimate of the source term $\mathcal{P}_{\varepsilon}^{A}$. We can rewrite, by definition of the tensor $\underline{\underline{\dot{e}}} \varepsilon$,

$$
\mathcal{P}_{\varepsilon}^{A}(t)=-\int_{\Omega_{0}} \underline{\underline{\Sigma}}^{A}: \underline{\underline{\dot{e}}}_{\varepsilon} \mathrm{d} \Omega_{0}=-\frac{1}{2} \int_{\Omega_{0}} \sigma_{a} \underline{\tau} \cdot \partial_{t}\left(\underline{\underline{F}}_{\varepsilon}^{T} \underline{\underline{F}}\right) \cdot \underline{\tau} \mathrm{d} \Omega_{0}
$$

Hence, by integrating over time, we find

$$
\int_{0}^{t} \mathcal{P}_{\varepsilon}^{A}(s) \mathrm{d} s=\frac{1}{2} \int_{0}^{t} \int_{\Omega_{0}} \partial_{t} \sigma_{a}(\underline{\xi}, s) I_{4, \varepsilon}(\underline{\xi}, s) \mathrm{d} \Omega_{0} \mathrm{~d} s-\frac{1}{2} \int_{\Omega_{0}} \sigma_{a}(\underline{\xi}, t) I_{4, \varepsilon}(\underline{\xi}, t) \mathrm{d} \Omega_{0},
$$

where $I_{4, \varepsilon}=\underline{\tau} \cdot\left(\underline{\underline{F}}_{\varepsilon}^{T} \cdot \underline{\underline{F}} \varepsilon\right) \cdot \underline{\tau}$. Hence, one can show using the Cauchy-Schwarz inequality that

$$
\begin{aligned}
\int_{0}^{t} \mathcal{P}_{\varepsilon}^{A}(s) \mathrm{d} s \leq \frac{\sup _{(\underline{\xi}, s) \in \Omega_{0} \times[0, T]}\left|\partial_{t} \sigma_{a}(\underline{\xi}, s)\right|}{2} \int_{0}^{t} & \int_{\Omega_{0}} I_{4, \varepsilon}(\underline{\xi}, s) \mathrm{d} \Omega_{0} \mathrm{~d} s \\
& +\frac{\sup _{(\underline{\xi}, s) \in \Omega_{0} \times[0, T]}\left|\sigma_{a}(\underline{\xi}, t)\right|}{2}\left|\Omega_{0}\right|^{\frac{1}{2}}\left(\int_{\Omega_{0}} I_{4, \varepsilon}^{2}(\underline{\xi}, t) \mathrm{d} \Omega_{0}\right)^{\frac{1}{2}} .
\end{aligned}
$$

Therefore, one can show that there exists a dimensionless scalar $C>0$ such that

$$
\int_{0}^{t} \mathcal{P}_{\varepsilon}^{A}(s) \mathrm{d} s \leq C\left(\sqrt{\mathcal{E}_{\varepsilon}^{t o t}(t)}+\int_{0}^{t} \varepsilon_{\varepsilon}^{t o t}(s) \mathrm{d} s\right)
$$


Application of the Gronwall inequality. We are now able to prove a bound on the total energy by an application of the Gronwall inequality. More precisely, we prove that the total internal energy $\varepsilon_{\varepsilon}^{\text {tot }}$ and the viscous contribution are bounded at every time $t \in[0, T]$, namely there exists a dimensionless scalar $C>0$ independent of $\varepsilon$ such that

$$
\mathcal{E}_{\varepsilon}^{t o t}(t)+\int_{0}^{t} \mathcal{P}_{\varepsilon}^{\mathrm{V}}(s) \mathrm{d} s \leq C \quad \forall t \in[0, T]
$$

For the sake of simplicity of exposure the value of $C$ will change along every line of the proof. Let us integrate with respect to time equation (A.2). We obtain

$$
\tilde{\mathcal{E}}_{\varepsilon}^{t o t}(t)=\int_{0}^{t} \mathcal{P}_{\varepsilon}^{\text {ext }}(s) \mathrm{d} s+\int_{0}^{t} \mathcal{P}_{\varepsilon}^{A}(s) \mathrm{d} s \quad \text { where } \quad \tilde{\mathcal{E}}_{\varepsilon}^{t o t}(t):=\mathcal{E}_{\varepsilon}^{t o t}(t)+\varepsilon^{-1} \int_{0}^{t} \mathcal{P}_{\varepsilon}^{\mathrm{V}}(s) \mathrm{d} s .
$$

Injecting Eq. (A.11) and Eq. (A.13) into (A.15), we obtain the energy estimate

$$
\varepsilon_{\varepsilon}^{t o t}(t)+(1-C \varepsilon) \int_{0}^{t} \mathcal{P}_{\varepsilon}^{\mathrm{V}}(s) \mathrm{d} s \leq C\left(1+\sqrt{\varepsilon_{\varepsilon}^{t o t}(t)}+\int_{0}^{t} \varepsilon_{\varepsilon}^{t o t}(s) \mathrm{d} s\right) .
$$

Then, using the Young inequality and assuming $\varepsilon$ small enough, one can show that

$$
\tilde{\varepsilon}_{\varepsilon}^{t o t}(t) \leq C\left(1+\int_{0}^{t} \tilde{\varepsilon}_{\varepsilon}^{t o t}(s) \mathrm{d} s\right)
$$

thus, by the Grönwall inequality in integral form, we can state that $\tilde{\mathcal{E}}_{\varepsilon}^{\text {tot }}(t) \leq C e^{C t}$.

Conclusion. From (A.14) and the definition of $\mathcal{E}_{\varepsilon}^{t o t}$ and $\mathcal{P}_{\varepsilon}^{V}$ we deduce that

$$
\begin{aligned}
\frac{\hat{\rho}_{0}}{2} \int_{\Omega_{0}}\left|\partial_{t} \underline{y}_{\varepsilon}\right|^{2} \mathrm{~d} \Omega_{0}+\int_{\Omega_{0}} & \left(W^{\mathrm{TI}}\left(\underline{\underline{e}}_{\varepsilon}\right)+8 \hat{\kappa}_{3} W^{\mathrm{NI}}\left(\underline{\underline{e}}_{\varepsilon}\right)\right) \mathrm{d} \Omega_{0} \\
& +\varepsilon^{-2}\left(1-8 \hat{\kappa}_{3} \varepsilon^{2}\right) \int_{\Omega_{0}} W^{\mathrm{NI}}\left(\underline{\underline{e}}_{\varepsilon}\right) \mathrm{d} \Omega_{0}+2 \varepsilon^{-1} \hat{\zeta} \int_{0}^{t} \int_{\Omega_{0}} \operatorname{tr}\left(\underline{\underline{C}}_{\varepsilon}^{-1} \cdot \underline{\underline{e}}_{\varepsilon}\right)^{2} \mathrm{~d} \Omega_{0} \leq C .
\end{aligned}
$$

Using Proposition A.2 we have

$$
-5 \hat{\kappa}_{3}\left|\Omega_{0}\right| \leq \int_{\Omega_{0}}\left(W^{\mathrm{TI}}\left(\underline{\underline{e}}_{\varepsilon}\right)+8 \hat{\kappa}_{3} W^{\mathrm{NI}}\left(\underline{\underline{e}}_{\varepsilon}\right)\right) \mathrm{d} \Omega_{0},
$$

hence, for all $\varepsilon \leq \varepsilon_{0}$ where $\varepsilon_{0}$ is such that $1-8 \hat{\kappa}_{3} \varepsilon_{0}^{2}=1 / 2$ we have

$$
\frac{\hat{\rho}_{0}}{2} \int_{\Omega_{0}}\left|\partial_{t} \underline{y}_{\varepsilon}\right|^{2} \mathrm{~d} \Omega_{0}+\frac{\varepsilon^{-2}}{2} \int_{\Omega_{0}} W^{\mathrm{NI}}\left(\underline{\underline{e}}_{\varepsilon}\right) \mathrm{d} \Omega_{0}+2 \varepsilon^{-1} \hat{\zeta} \int_{0}^{t} \int_{\Omega_{0}} \operatorname{tr}\left(\underline{\underline{C}}_{\varepsilon}^{-1} \cdot \underline{\underline{e}}_{\varepsilon}\right)^{2} \mathrm{~d} \Omega_{0} \leq C,
$$

which concludes the proof of Lemma 2.1. 


\section{Appendix B Full expression of a linearised stress tensor term}

We define the transversely isotropic potential associated to the displacement $\underline{y}_{0}$ as

$$
W_{0}^{\mathrm{TI}}\left(\underline{\underline{e}}_{0}\right)=\hat{\kappa}_{1} e^{\hat{\eta}_{1}\left(I_{1,0}-3\right)^{2}}+\hat{\kappa}_{2} e^{\hat{\eta}_{2}\left(I_{4,0}-1\right)^{2}}+\hat{\kappa}_{3}\left(I_{2,0}-2 \log \left(J_{0}^{2}\right)-3\right)
$$

with $\underline{\underline{e}}_{0}=\left(\underline{\underline{F}}_{0}^{T} \cdot \underline{\underline{F}}_{0}-\underline{\underline{\mathbb{1}}}\right) / 2$ and

$$
I_{1,0}=3+2 \operatorname{tr}\left(\underline{\underline{e}}_{0}\right), \quad I_{4,0}=1+2 \underline{\tau} \cdot \underline{\underline{e}}_{0} \cdot \underline{\tau}, \quad I_{2,0}=\left|\operatorname{adj} \underline{\underline{F}}_{0}\right|^{2} .
$$

The corresponding stress tensor is given by

$$
\underline{\underline{\Sigma}}_{0}^{\mathrm{TI}}=\frac{\partial W_{0}^{\mathrm{TI}}}{\partial \underline{\underline{e}}_{0}}=K_{1,0}\left(I_{1,0}-3\right) \underline{\underline{\mathbb{1}}}+K_{4,0}\left(I_{4,0}-1\right) \underline{\tau} \otimes \underline{\tau}+2 \hat{\kappa}_{3}\left(I_{1,0} \underline{\underline{\mathbb{1}}}-\underline{\underline{C}}_{0}-2 \underline{\underline{C}}_{0}^{-1}\right),
$$

with

$$
K_{1,0}:=4 \hat{\kappa}_{1} \hat{\eta}_{1} e^{\hat{\eta}_{1}\left(I_{1,0}-3\right)^{2}}, \quad K_{4,0}:=4 \hat{\kappa}_{2} \hat{\eta}_{2} e^{\hat{\eta}_{2}\left(I_{4,0}-1\right)^{2}} .
$$

One can observe that $\underline{\underline{\Sigma}}_{0}^{\mathrm{TI}}$ vanishes if $\underline{\underline{e}}_{0}=\underline{\underline{0}}$. Furthermore, the full definition of the tensor $\underline{\underline{\mathbf{A}}}_{0}$ is given, for any test function $\underline{w}$, by

$$
\begin{aligned}
\underline{\underline{\mathbf{A}}}_{0}: \underline{\underline{e}}_{0}(\underline{w})= & \frac{\partial \underline{\underline{\Sigma}}_{0}^{\mathrm{TI}}}{\partial \underline{\underline{e}}_{0}}: \underline{\underline{e}}_{0}(\underline{w}) \\
& =Q_{1,0} \operatorname{tr}\left(\underline{\underline{e}}_{0}(\underline{w})\right) \underline{\underline{\mathbb{1}}}+Q_{4,0}\left(\underline{\tau} \cdot \underline{\underline{e}}_{0}(\underline{w}) \cdot \underline{\tau}\right) \underline{\tau} \otimes \underline{\tau}+4 \hat{\kappa}_{3}\left(2 \underline{\underline{C}}_{0}^{-1} \cdot \underline{\underline{e}}_{0}(\underline{w}) \cdot \underline{\underline{C}}_{0}^{-1}-\underline{\underline{e}}_{0}(\underline{w})\right)
\end{aligned}
$$

with

$$
Q_{1,0}=2 K_{1,0}\left(1+2 \hat{\eta}_{1}\left(I_{1,0}-3\right)^{2}\right)+4 \hat{\kappa}_{3}, \quad Q_{4,0}=2 K_{4,0}\left(1+2 \hat{\eta}_{1}\left(I_{4,0}-1\right)^{2}\right) .
$$

If $\underline{\underline{e}}_{0}=\underline{\underline{0}}$, then

$$
\underline{\underline{\mathbf{A}}}_{0}: \underline{\underline{e}}_{0}(\underline{w})=\underline{\underline{\mathbf{A}}}_{0}: \underline{\underline{\varepsilon}}(\underline{w})=\left(8 \hat{\kappa}_{1} \hat{\eta}_{1}+4 \hat{\kappa}_{3}\right) \operatorname{tr}(\underline{\underline{\varepsilon}}(\underline{w})) \underline{\underline{\mathbb{1}}}+8 \hat{\kappa}_{2} \hat{\eta}_{2}(\underline{\tau} \cdot \underline{\underline{\varepsilon}}(\underline{w}) \cdot \underline{\tau}) \underline{\tau} \otimes \underline{\tau}+4 \hat{\kappa}_{3} \underline{\underline{\varepsilon}}(\underline{w}),
$$

with $\underline{\underline{\varepsilon}}(\underline{w})=\underline{\underline{\nabla}} \underline{w}+(\underline{\underline{\nabla}} \underline{w})^{T}$. The expression above shows that $\underline{\underline{\mathbf{A}}}_{0}$ is a positive fourth-order tensor for small values of $\underline{\underline{e}}_{0}$. Indeed, for all symmetric second-order tensor $\underline{\underline{\varepsilon}}$, we have

$$
\left(\underline{\underline{\mathbf{A}}}_{0}: \underline{\underline{\varepsilon}}\right): \underline{\underline{\varepsilon}}=\left(8 \hat{\kappa}_{1} \hat{\eta}_{1}+4 \hat{\kappa}_{3}\right) \operatorname{tr}(\underline{\underline{\varepsilon}})^{2}+8 \hat{\kappa}_{2} \hat{\eta}_{2}(\underline{\tau} \cdot \underline{\underline{\varepsilon}} \cdot \underline{\tau})^{2}+4 \hat{\kappa}_{3} \underline{\underline{\varepsilon}}: \underline{\underline{\varepsilon}} \geq 4 \hat{\kappa}_{3}|\underline{\underline{\varepsilon}}|^{2} .
$$




\section{References}

[1] Armen P Sarvazyan, Oleg V Rudenko, Scott D Swanson, J Brian Fowlkes, and Stanislav Y Emelianov. Shear wave elasticity imaging: a new ultrasonic technology of medical diagnostics. Ultrasound in medicine $\&$ biology, 24(9):1419-1435, 1998.

[2] Mostafa Fatemi and James F Greenleaf. Ultrasound-stimulated vibro-acoustic spectrography. Science, 280(5360):82-85, 1998.

[3] Kathryn Nightingale, Stephen McAleavey, and Gregg Trahey. Shear-wave generation using acoustic radiation force: in vivo and ex vivo results. Ultrasound in medicine $\&$ biology, 29(12):1715-1723, 2003.

[4] Jérémy Bercoff, Mickael Tanter, and Mathias Fink. Supersonic shear imaging: a new technique for soft tissue elasticity mapping. IEEE transactions on ultrasonics, ferroelectrics, and frequency control, 51(4):396-409, 2004.

[5] Stephen A McAleavey, Manoj Menon, and Jarrod Orszulak. Shear-modulus estimation by application of spatially-modulated impulsive acoustic radiation force. Ultrasonic imaging, 29(2):87-104, 2007.

[6] Pengfei Song, Heng Zhao, Armando Manduca, Matthew W Urban, James F Greenleaf, and Shigao Chen. Comb-push ultrasound shear elastography (CUSE): a novel method for two-dimensional shear elasticity imaging of soft tissues. IEEE transactions on medical imaging, 31(9):1821-1832, 2012.

[7] GR Torr. The acoustic radiation force. American Journal of Physics, 52:402-408, 1984.

[8] Kathryn Nightingale, Mary Scott Soo, Roger Nightingale, and Gregg Trahey. Acoustic radiation force impulse imaging: in vivo demonstration of clinical feasibility. Ultrasound in medicine $\mathbb{E}$ biology, 28(2):227-235, 2002.

[9] Armen P Sarvazyan, Oleg V Rudenko, and Wesley L Nyborg. Biomedical applications of radiation force of ultrasound: historical roots and physical basis. Ultrasound in medicine $\mathscr{G}$ biology, 36(9):1379$1394,2010$.

[10] Armen Sarvazyan, Timothy J Hall, Matthew W Urban, Mostafa Fatemi, Salavat R Aglyamov, and Brian S Garra. An overview of elastography-an emerging branch of medical imaging. Current medical imaging reviews, 7(4):255-282, 2011.

[11] OV Rudenko, AP Sarvazyan, and S Yu Emelianov. Acoustic radiation force and streaming induced by focused nonlinear ultrasound in a dissipative medium. The Journal of the Acoustical Society of America, 99(5):2791-2798, 1996.

[12] Mark F Hamilton, David T Blackstock, et al. Nonlinear acoustics, volume 1. Academic press San Diego, 1998. 
[13] Evgenia A. Zabolotskaya, Mark F. Hamilton, Yurii A. Ilinskii, and G. Douglas Meegan. Modeling of nonlinear shear waves in soft solids. The Journal of the Acoustical Society of America, 116(5):2807$2813,2004$.

[14] J-L Gennisson, Mathieu Rénier, Stefan Catheline, Christophe Barrière, Jeremy Bercoff, Mickael Tanter, and Mathias Fink. Acoustoelasticity in soft solids: Assessment of the nonlinear shear modulus with the acoustic radiation force. The Journal of the Acoustical Society of America, 122(6):3211-3219, 2007.

[15] EV Dontsov and Bojan B Guzina. Effect of low-frequency modulation on the acoustic radiation force in newtonian fluids. SIAM Journal on Applied Mathematics, 71(1):356-378, 2011.

[16] Egor V Dontsov and Bojan B Guzina. Acoustic radiation force in tissue-like solids due to modulated sound field. Journal of the Mechanics and Physics of Solids, 60(10):1791-1813, 2012.

[17] Egor V Dontsov and Bojan B Guzina. On the kzk-type equation for modulated ultrasound fields. Wave Motion, 50(4):763-775, 2013.

[18] Lev Ostrovsky, Alexander Sutin, Yuri Il'inskii, Oleg Rudenko, and Armen Sarvazyan. Radiation force and shear motions in inhomogeneous media. The Journal of the Acoustical Society of America, 121(3):1324-1331, 2007.

[19] Mark L Palmeri, Amy C Sharma, Richard R Bouchard, Roger W Nightingale, and Kathryn R Nightingale. A finite-element method model of soft tissue response to impulsive acoustic radiation force. IEEE transactions on ultrasonics, ferroelectrics, and frequency control, 52(10):1699-1712, 2005.

[20] Annette Caenen. A biomechanical analysis of shear wave elastography in pediatric heart models. $\mathrm{PhD}$ thesis, Ghent University, 2018.

[21] Dominique Chapelle, Patrick Le Tallec, Philippe Moireau, and Michel Sorine. An energy-preserving muscle tissue model: formulation and compatible discretizations. International Journal for Multiscale Computational Engineering, 10(2):189-211, 2012.

[22] Lawrence E Malvern. Introduction to the Mechanics of a Continuous Medium. Number Monograph. 1969.

[23] Philippe G Ciarlet. Mathematical elasticity. vol. i, volume 20 of studies in mathematics and its applications, 1988.

[24] Raymond W Ogden. Non-linear elastic deformations. Courier Corporation, 1997.

[25] Gerhard A Holzapfel and Ray W Ogden. Constitutive modelling of passive myocardium: a structurally based framework for material characterization. Philosophical Transactions of the Royal Society of London A: Mathematical, Physical and Engineering Sciences, 367(1902):3445-3475, 2009. 
[26] Myrianthi Hadjicharalambous, Liya Asner, Radomir Chabiniok, Eva Sammut, James Wong, Devis Peressutti, Eric Kerfoot, Andrew King, Jack Lee, Reza Razavi, et al. Non-invasive model-based assessment of passive left-ventricular myocardial stiffness in healthy subjects and in patients with non-ischemic dilated cardiomyopathy. Annals of biomedical engineering, 45(3):605-618, 2017.

[27] Philippe G Ciarlet and Giuseppe Geymonat. Sur les lois de comportement en élasticité non linéaire compressible. CR Acad. Sci. Paris Sér. II, 295:423-426, 1982.

[28] Federica Caforio. Mathematical modelling and numerical simulation of elastic wave propagation in soft tissues with application to cardiac elastography. PhD thesis, Université Paris-Saclay, 2019.

[29] Annette Caenen, Mathieu Pernot, Mathias Peirlinck, Luc Mertens, Abigail Swillens, and Patrick Segers. An in silico framework to analyze the anisotropic shear wave mechanics in cardiac shear wave elastography. Physics in Medicine ES Biology, 63(7):075005, 2018.

[30] Osman Gültekin, Gerhard Sommer, and Gerhard A Holzapfel. An orthotropic viscoelastic model for the passive myocardium: continuum basis and numerical treatment. Computer methods in biomechanics and biomedical engineering, 19(15):1647-1664, 2016.

[31] Jacques-Louis Lions, George Papanicolaou, and Alain Bensoussan. Asymptotic analysis for periodic structures. North-Holland, 1978. 